\title{
Thermodynamically constrained averaging theory approach for modeling flow and transport phenomena in porous medium systems: 3. Single-fluid-phase flow
}

\author{
William G. Gray, ${ }^{*}$ Cass T. Miller \\ Department of Environmental Sciences and Engineering, University of North \\ Carolina, Chapel Hill, North Carolina 27599-7431, USA
}

\begin{abstract}
This work is the third in a series of papers on the thermodynamically constrained averaging theory (TCAT) approach to modeling flow and transport phenomena in multiscale porous medium systems. Building upon the general TCAT framework and the mathematical foundation presented in previous works in this series, we demonstrate the TCAT approach for the case of single-fluid-phase flow. The formulated model is based upon conservation equations for mass, momentum, and energy and a general entropy inequality constraint, which is developed to guide model closure. A specific example of a closed model is derived under limiting assumptions using a linearization approach and these results are compared and contrasted with the traditional single-phase-flow model. Potential extensions to this work are discussed. Specific advancements in this work beyond previous averaging theory approaches to single-phase flow include use of macroscale thermodynamics that is averaged from the microscale, the use of derived equilibrium conditions to guide a flux-force pair
\end{abstract}


approach to simplification, use of a general Lagrange multiplier approach to connect conservation equation constraints to the entropy inequality, and a focus on producing complete, closed models that are solvable.

Key words: Porous medium models, Averaging theory, TCAT, Single-phase flow

* Corresponding author

Email addresses: GrayWG@unc.edu (William G. Gray,), casey_miller@unc.edu

(Cass T. Miller). 


\section{Notation}

Roman letters

$b \quad$ external entropy source per unit volume

C Greens' deformation tensor

$\hat{c} \quad$ compressibility parameter

d rate of strain tensor

E internal energy per unit volume

$\hat{E} \quad$ Young's modulus

$E_{T} \quad$ total energy per unit volume

$\mathcal{E} \quad$ the set of entities in the model

$\mathcal{E} \quad$ conservation of energy equation

$\mathcal{E}_{\mathrm{c}} \quad$ connected set of entities

$\hat{E}_{\nu} \quad$ variable grouping defined by eqn (114)

e solid-phase Eulerian strain tensor

$e_{z z}^{s} \quad$ macroscale solid-phase Eulerian strain tensor diagonal component

for the vertical direction

F thermodynamic force tensor

F thermodynamic force vector

F thermodynamic force scalar

$\mathcal{F} \quad$ set of all thermodynamic forces

G geometric orientation tensor for an interface

g gravitational acceleration vector

$g$ magnitude of gravitational acceleration

$H \quad$ hydraulic head

$h \quad$ heat source per unit volume 


\begin{tabular}{|c|c|}
\hline 1 & identity tensor \\
\hline $\mathbf{I}^{\prime}$ & surface identity tensor \\
\hline $\mathcal{J}$ & index set of entities \\
\hline$J_{c}$ & index set of connected entities \\
\hline $\mathrm{J}_{p}$ & index set of phase entities \\
\hline J & thermodynamic flux tensor \\
\hline $\mathbf{J}$ & thermodynamic flux vector \\
\hline$J$ & thermodynamic flux scalar \\
\hline J & set to all thermodynamic fluxes \\
\hline j & solid-phase Jacobian \\
\hline$\hat{\mathbf{K}}$ & hydraulic conductivity tensor \\
\hline$\hat{\mathbf{K}}_{q}$ & $\begin{array}{l}\text { second-rank, symmetric, positive semi-definite heat conduction ten- } \\
\text { sor }\end{array}$ \\
\hline$K_{E}$ & kinetic energy per unit mass due to microscale velocity fluctuations \\
\hline$\hat{K}_{S}$ & bulk modulus of the solid phase \\
\hline$\hat{K}_{T}$ & bulk modulus of the skeleton \\
\hline k & unit Cartesian vector oriented vertically upward \\
\hline$\hat{k}_{m}$ & non-negative interfacial mass transer parameter \\
\hline$\hat{k}_{q}$ & non-negative interfacial heat transfer parameter \\
\hline $\mathcal{M}$ & conservation of mass equation \\
\hline$\stackrel{\kappa \rightarrow}{M}$ & transfer of mass from the $\kappa$ to the $\iota$ entity \\
\hline$\stackrel{\kappa \rightarrow l}{M}_{E}^{\iota}$ & $\begin{array}{l}\text { transfer of energy from the } \kappa \text { to the } \iota \text { entity resulting from mass } \\
\text { transfer }\end{array}$ \\
\hline$\stackrel{\kappa}{\mathbf{M}}_{v}^{l}$ & $\begin{array}{l}\text { exchange of momentum from the } \kappa \text { to the } \iota \text { entity resulting from } \\
\text { mass transfer }\end{array}$ \\
\hline$\stackrel{\kappa}{M} M_{\eta}^{l}$ & $\begin{array}{l}\text { exchange of entropy from the } \kappa \text { to the } \iota \text { entity resulting from mass } \\
\text { transfer }\end{array}$ \\
\hline
\end{tabular}




\begin{tabular}{|c|c|}
\hline $\mathcal{P}$ & conservation of momentum equation \\
\hline $\mathcal{P}_{i}$ & general microscale property \\
\hline$p$ & fluid pressure \\
\hline$\stackrel{\kappa \rightarrow \iota}{Q}$ & $\begin{array}{l}\text { transfer of energy from the } \kappa \text { to the } \iota \text { entity resulting from phase } \\
\text { change, interfacial stress, and heat transfer }\end{array}$ \\
\hline q & heat flux vector \\
\hline$\hat{\mathbf{R}}$ & $\begin{array}{l}\text { symmetric, positive semi-definite second-rank momentum resistance } \\
\text { tensor }\end{array}$ \\
\hline $\mathcal{S}$ & entropy balance equation \\
\hline$\hat{S}_{s}$ & specific storage coefficient \\
\hline $\mathcal{T}$ & $\begin{array}{l}\text { CIT-based thermodynamic equation for material derivative of inter- } \\
\text { nal energy }\end{array}$ \\
\hline$\stackrel{\kappa \rightarrow \iota}{\mathbf{T}}$ & transfer of momentum from the $\kappa$ to the $\iota$ entity \\
\hline$\stackrel{\kappa \rightarrow \iota}{T_{v}^{\iota}}$ & $\begin{array}{l}\text { transfer of energy from the } \kappa \text { to the } \iota \text { entity resulting from interfacial } \\
\text { stress }\end{array}$ \\
\hline $\mathbf{t}$ & stress tensor \\
\hline$t$ & time \\
\hline $\mathcal{V}$ & the set of unknown variables requiring closure relations \\
\hline $\mathbf{v}$ & velocity vector \\
\hline $\mathbf{v}^{\bar{l}, \bar{s}}$ & $\begin{array}{l}\text { mass-averaged velocity of the } \iota \text { entity relative to the mass-averaged } \\
\text { velocity of the } s \text { entity }\end{array}$ \\
\hline$w$ & weighting function in averaging operator \\
\hline $\mathrm{X}$ & position vector in the solid phase initially \\
\hline $\mathbf{x}$ & position vector in the solid phase \\
\hline
\end{tabular}


Greek letters

$\hat{\alpha} \quad$ biot coefficient

$\hat{\beta} \quad$ compressibility parameter

$\Gamma \quad$ boundary of domain of interest

$\gamma \quad$ interfacial tension

$\epsilon^{\iota} \quad$ specific measure of the $\iota$ entity

$\eta \quad$ entropy per unit volume

$\theta \quad$ temperature

$\Lambda \quad$ entropy production per unit volume

$\boldsymbol{\lambda} \quad$ vector of Lagrange multipliers

$\lambda \quad$ Lagrange multiplier

$\mu \quad$ chemical potential

$\hat{\nu} \quad$ Poisson's ratio

$\rho \quad$ density

$\boldsymbol{\sigma} \quad$ solid-phase Lagrangian stress tensor

$\boldsymbol{\tau} \quad$ effective solid-phase stress tensor

$\stackrel{\kappa \rightarrow \iota}{\Phi} \quad$ transfer of entropy from the $\kappa$ to the $\iota$ entity

$\varphi \quad$ entropy flux vector

$\psi \quad$ gravitational potential

$\Omega \quad$ spatial domain

$\bar{\Omega} \quad$ closed domain

Subscripts and superscripts

b bulk qualifier (superscript)

D material derivative equivalence qualifier (subscript) 


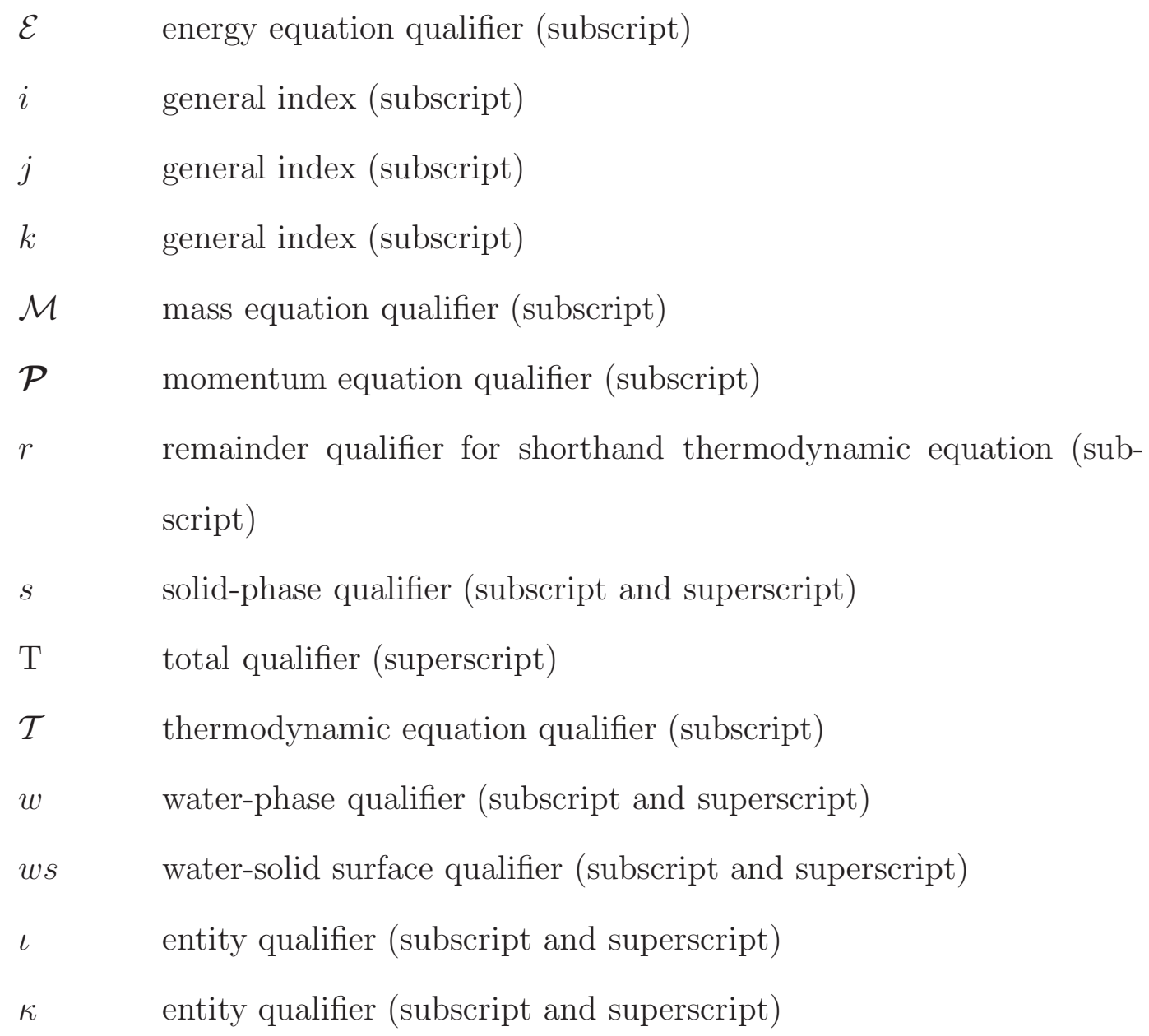

\section{Abbreviations}

$\begin{array}{ll}\text { AEI } & \text { augmented entropy inequality } \\ \text { CEI } & \text { constrained entropy inequality } \\ \text { CIT } & \text { classical irreversible thermodynamics } \\ \text { EI } & \text { entropy inequality } \\ \text { EPP } & \text { entropy production postulate } \\ \text { REV } & \text { representative elementary volume } \\ \text { SEI } & \text { simplified entropy inequality } \\ \text { TCAT } & \text { thermodynamically constrained averaging theory }\end{array}$




\section{Introduction}

This paper is the third in a series of efforts designed to yield complete, rigorous, closed models that describe transport phenomena in multiscale porous medium systems using the thermodynamically constrained averaging theory (TCAT) approach. Work to date has outlined a general TCAT approach that can be used to generate such models [20] and laid a mathematical foundation upon which these models can be constructed [25]. We will build on these results in the current and subsequent papers to construct closed models for important systems, to compare and contrast these models with conventional models in use for similar purposes, and to compare these new models with highly resolved sub-scale simulations and experimental observations. The present paper is focused on single-fluid-phase flow in porous media. This system has been chosen because it provides a relatively simple setting in which to demonstrate the application of the TCAT approach and to illustrate that putting even this well-studied system on firm theoretical footing illuminates some important intrinsic assumptions in conventional models.

The traditional model for single-phase flow is derived typically by (1) writing an equation of mass conservation for a fluid phase; (2) using Darcy's law as an approximate momentum equation to remove the superficial velocity vector from the conservation equation; (3) assuming a simple equation of state for the fluid phase that relates its density and pressure; (4) assuming that spatial

gradients in density are small; and (5) approximating the compressibilities of the fluid and solid phases in time using linear, reversible compressibility theory. Derivation of the standard single-phase model for flow through porous media along these lines is routine, and the resultant model is used nearly universally. 
Today, the physics of single-fluid-phase (hereinafter, simply single-phase) flow through porous media is considered well-established [e.g. 3, 4, 11, 12, 14]. Popular, well-documented, and widely used numerical simulators that solve a traditional model for single-phase flow for transient conditions in three spatial dimensions have existed for 18 years [24].

Efforts have been undertaken to provide a more comprehensive and satisfying theoretical basis for modeling single-phase flow than the traditional approach. One way in which this has been accomplished is by deriving Darcy's law from first principles. Darcy $[9,10]$ performed a careful series of one-dimensional column experiments to measure head loss through homogeneous sand systems, and Darcy's law was inferred from these experiments; it has since been a cornerstone principle for flow through porous materials. In recent years, a variety of approaches have been advanced to derive Darcy's law based on describing flow through a porous medium at the microscale using the Stokes equations or the Navier-Stokes equations and upscaling this description to the macroscale [e.g. 2, 26-30]. Such efforts have provided a mathematical route to Darcy's law, but not a comprehensive, thermodynamically constrained theory yielding flow equations appropriate for more complex situations, such as cases where the Darcian linear proportionality between velocity and the potential gradient does not apply or single-phase flow through deformable, non-isothermal porous media. Furthermore, inconsistencies related to the original form of Darcy's law and its common usage have recently been discussed even for very simple systems [19]. In the 150 years since its formulation, Darcy's law has undergone a series of extensions such that it is now routinely applied in a variety of settings not supported by the original set of experiments, including anisotropic conditions and the flow of multiple fluid phases. 
Formal averaging approaches, which are a central component of this work, have been developed over the last three decades and applied to formulate models for both single-fluid-phase and multiple-fluid-phase systems. Formal averaging methods provide a means to not only show conditions under which Darcy's law emerges as an approximation of a more general theory, but also to examine a wide range of cases for which the traditional single-phase model does not provide a description of sufficient accuracy. Previous work in this series reviewed averaging theory methods and highlighted the limitations that motivate the TCAT approach [20].

The overall goal of this work is to formulate a rigorous, first-principles-based theory for single-phase flow in porous media for which the underlying assumptions are explicitly indicated. The specific objectives of this work are: (1) to develop a general, thermodynamically consistent theory for single-phase flow based upon well defined macroscale variables and a clear connection to the microscale; (2) to provide an example of how a simple single-phase model can be deduced based upon the general theory; (3) to compare the conventionallyemployed single-phase model with a model that emerges here from the general theory; and (4) to suggest a new set of single-phase model extensions that may be derived from the general theory.

This work differs from previous averaging theory work in several respects including: (1) thermodynamic constraints are developed by averaging classical irreversible thermoynamics from the microscale to the macroscale; (2) equilibrium conditions are summarized that provide firm guidance for model formulation; (3) a flux-force pair approach is used to develop and simplify the entropy inequality using the equilibrium conditions; (4) a general Lagrange multiplier approach is used to connect macroscale conservation equations to the entropy 
inequality and solved in light of solvability constraints; (5) a clear separation between exact forms of the general model formulation and approximate forms are noted; (6) a detailed set of assumptions are made and the details of a complete, closed model formulation are presented; and (7) the general results achieved provide a means to develop many alternative models based upon other sets of assumptions and applying to more complicated cases than the simple traditional single-phase flow model. The amount of work needed to accomplish these tasks is not inconsequential. We therefore wish to stress that this paper is a derivation of general conservation equations for single-phase flow, not a narrowly targeted set of steps aimed at obtaining Darcy's law. The starting point for the upscaling is the general conservation equations for the fluid and the solid along with an established thermodynamic theory. This starting point, coupled with the systematic framework provided by the TCAT approach allows us to gain new insights into the mechanisms of single-phase flow systems and also sets the stage for analyses of more complex systems in subsequent installments.

\section{General approach}

The macroscale system considered in this work is shown schematically in Fig. 1 and consists of a macroscale domain, $\Omega$; a volume occupied by a solid phase, $\Omega_{s}$; a volume occupied by the fluid phase, $\Omega_{w}$; and an interfacial region between the solid and fluid phases, $\Omega_{w s}$. The notion of writing specific conservation equations for an interface is a point of departure from the development of the traditional model, where conservation of mass equations are written only for the phases. Interface equations are written to account for system properties, 
and their variation in time and space, at the region of transition from one phase to another. Molecular interactions that occur at such interfaces lead to a change in most physicochemical properties of concern over short length scales in the vicinity of the surface. Inclusion of conservation equations for interfaces allows deviations from the mean bulk behavior in the transition regions to be properly modeled. An important result from this approach is that evolution equations for interfacial areas follow from the general theory.

Fig. 1. Single-fluid-phase system.

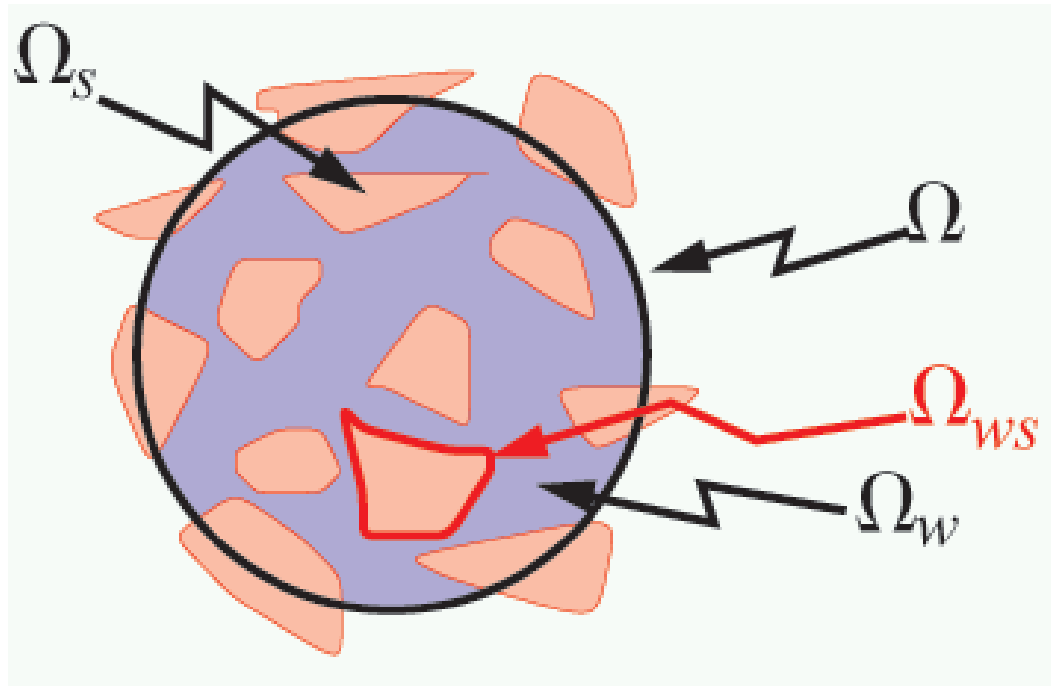

A macroscale system is one whose length scale is sufficiently large that it includes elements of all entities (i.e., phases and interfaces). The model is formulated in terms of averages of microscale properties over a representative elementary volume (REV) with this length scale, but such models do not require detailed knowledge of the microscale pore structure. However, we do seek a formulation that explicitly defines macroscale variables in terms of welldefined quantities arising in a microscale description. Such an approach is unambiguous and provides a means for connecting microscale and macroscale descriptions. Conditions for which a well-defined REV does not exist occur routinely in nature but are outside the scope of this investigation. 
Rigorous macroscale models for single-phase systems can be developed based upon the TCAT approach [20], which has the following steps:

(1) an entropy inequality (EI) expression for the entire system of concern is generated;

(2) an appropriate set of mass, momentum, and energy conservation equations is formulated at the desired scale for all relevant entities (phase volumes and interfaces) based upon clearly defined averages of microscale quantities;

(3) an appropriate microscale thermodynamic theory is averaged up to the desired scale, and differential forms of internal energy dependence for spatial and temporal derivatives are generated;

(4) the EI is augmented using the product of Lagrange multipliers with conservation equations and differential, consistent-scale thermodynamic equations;

(5) the set of Lagrange multipliers is determined to select the combination of conservation equations that describes the physics of interest and to eliminate time derivatives from the augmented EI (AEI) producing the constrained EI (CEI);

(6) geometric identities and approximations are applied to the CEI to eliminate additional remaining time derivatives as needed to produce the simplified EI (SEI);

(7) the resultant SEI is used to guide the formulation of general forms of closure approximations consistent with the second law of thermodynamics; and

(8) microscale and macroscale modeling and experimentation are used to advance appropriate forms of closure relations. 
Within this general approach, notions related to the scales of concern and fundamental definitions upon which this general theory is based, which we term the standard averaging definitions, are detailed in Miller and Gray [25]. The general TCAT approach, which can be applied across a wide range of space and time scales, is detailed in Gray and Miller [20]. The specific macroscopic, single-phase flow application of concern in this work is an example application of this general theory. The following sections correspond to the general TCAT approach summary given above and detail the development of a complete closed single-phase model.

\section{$3 \quad$ Entropy Inequality}

In recent work, macroscale conservation equations for mass, momentum, energy, and entropy for phase volumes, interfaces, common curves, and common points that can exist within a multiphase system have been developed [17] and employed to obtain models for two-phase flow [15, 22]. We refer to the various regions (phase volumes, interfaces, common curves, and common points) as entities. Membership in the set of entities depends upon the system under consideration. For the single-fluid system of primary concern here, the set of entities in an REV, and assumed to be at this scale, is given by

$$
\mathcal{E}=\left\{\Omega_{\iota} \mid \iota \in \mathcal{J}\right\}=\left\{\Omega_{w}, \Omega_{s}, \Omega_{w s}\right\}
$$

where the index set of entity qualifiers is given by

$$
\mathcal{J}=\{w, s, w s\}
$$


where $w$ represents the water phase, $s$ the solid phase, and $w s$ the water-solid interface.

We also introduce the notion of a connected entity set, which we define for entity $\mathcal{E}_{\iota}$ as

$$
\mathcal{E}_{\mathrm{c} \iota}=\left\{\Omega_{\kappa} \mid\left(\bar{\Omega}_{\iota} \cap \bar{\Omega}_{\kappa} \neq \emptyset\right) \wedge\left(\bar{\Omega}_{\iota} \neq \bar{\Omega}_{\kappa}\right), \forall \Omega_{\kappa} \in \mathcal{E}\right\}
$$

where the closure of the entities is defined as $\bar{\Omega}_{\iota}=\Omega_{\iota} \cup \Gamma_{\iota}, \Gamma_{\iota}$ is the boundary of $\Omega_{\iota}$, and $\mathcal{J}_{c \iota}$ is the index set corresponding to $\mathcal{E}_{\mathrm{c} \iota}$ so $\mathcal{E}_{c \iota}=\left\{\Omega_{\kappa} \mid \kappa \in \mathcal{J}_{\mathrm{c} \iota}\right\}$. The connected entity set for entity $\iota$ is thus the set of entities with which it has direct contact. The three instances of connected entity sets relevant for the single-phase flow problem of concern in this work are

$$
\begin{aligned}
\mathcal{E}_{\mathrm{c} w} & =\left\{\Omega_{w s}\right\} \\
\mathcal{E}_{\mathrm{cs}} & =\left\{\Omega_{w s}\right\} \\
\mathcal{E}_{\mathrm{c} w s} & =\left\{\Omega_{w}, \Omega_{s}\right\}
\end{aligned}
$$

where $\mathcal{J}_{c w}, \mathcal{J}_{c s}$, and $\mathcal{J}_{c w s}$ are the connected index sets that correspond to the respective connected entity sets, e.g., $\mathcal{J}_{c w}=\{w s\}$. The use of set notation for entities, connected entities, and index sets provides a means to develop a general, compact notation that will prove useful for generating TCAT models for a variety of systems.

A general balance equation is needed for entropy associated with the $\iota$ entity at the macroscale. This equation can be derived by averaging from the microscale to the macroscale or by using a localization approach to obtain

$$
\frac{\mathrm{D}^{\bar{\iota}} \eta^{\overline{\bar{\iota}}}}{\mathrm{D} t}+\eta^{\overline{\bar{\iota}}} \mathbf{I}: \mathbf{d}^{\overline{\bar{\iota}}}-\nabla \cdot\left(\epsilon^{\iota} \boldsymbol{\varphi}^{\overline{\bar{\iota}}}\right)-\epsilon^{\iota} b^{\iota}-\sum_{\kappa \in \mathcal{J}_{c \iota}}\left(\stackrel{\kappa \rightarrow \iota}{M_{\eta}^{\iota}}+\stackrel{\kappa \rightarrow \iota}{\Phi}\right)=\Lambda^{\overline{\bar{\iota}}}, \quad \text { for } \iota \in \mathcal{J}
$$

Quantities in this equation have been obtained by making use of the averaging 
operator [25]:

$$
\left\langle\mathcal{P}_{i}\right\rangle_{\Omega_{j}, \Omega_{k}, w}=\frac{\int_{\Omega_{j}} w \mathcal{P}_{i} \mathrm{~d} \mathfrak{r}}{\int_{\Omega_{k}} w \mathrm{~d} \mathfrak{r}}
$$

where $\mathcal{P}_{i}$ is a general property to be averaged; and $w$ is a weighting function. When $w=1, w$ is omitted as a subscript on the left side of eqn (8). The terms in eqn (7) are defined in terms of the averaging operator as

$$
\begin{aligned}
\eta^{\overline{\bar{t}}} & =\left\langle\eta_{\iota}\right\rangle_{\Omega_{\iota}, \Omega} \\
\mathbf{v}^{\bar{\tau}} & =\left\langle\mathbf{v}_{\iota}\right\rangle_{\Omega_{\iota}, \Omega_{\iota}, \rho_{\iota}} \\
\mathbf{d}^{\overline{\bar{\tau}}} & =\frac{1}{2}\left[\nabla \mathbf{v}^{\bar{\imath}}+\left(\nabla \mathbf{v}^{\bar{\tau}}\right)^{\mathrm{T}}\right] \\
\boldsymbol{\varphi}^{\overline{\bar{t}}} & =\left\langle\boldsymbol{\varphi}_{\iota}\right\rangle_{\Omega_{\iota}, \Omega_{\iota}}-\left\langle\eta_{\iota}\left(\mathbf{v}_{\iota}-\mathbf{v}^{\bar{\tau}}\right)\right\rangle_{\Omega_{\iota}, \Omega_{\iota}} \\
b^{\iota} & =\left\langle b_{\iota}\right\rangle_{\Omega_{\iota}, \Omega_{\iota}} \\
\Lambda^{\overline{\bar{l}}} & =\left\langle\Lambda_{\iota}\right\rangle_{\Omega_{\iota}, \Omega}
\end{aligned}
$$

and the material derivative is defined as

$$
\frac{\mathrm{D}^{\bar{\imath}}}{\mathrm{D} t}=\frac{\partial}{\partial t}+\mathbf{v}^{\bar{\imath}} \cdot \nabla
$$

where subscripted quantities are microscale quantities, superscripted quantities are macroscale quantities, $\eta^{\overline{\bar{\tau}}}$ is the entropy of entity $\iota$ per unit REV; $t$ is time; $\mathbf{I}$ is the identity tensor; $\mathbf{d}^{\overline{\bar{t}}}$ is the rate of strain tensor; $\mathbf{v}^{\bar{\tau}}$ is a mass averaged intrinsic velocity; $\epsilon^{\iota}$ is the specific entity measure for the $\iota$ entity, which is a volume fraction for phase entities or a specific interfacial area for the interface entity; $\varphi^{\overline{\bar{t}}}$ is an entropy flux vector; $b^{\iota}$ is an entropy source per unit volume; $\stackrel{\kappa \rightarrow}{M}$. accounts for entropy exchange from the $\kappa$ entity to the $\iota$ entity accompanying mass transfer between these entities; $\stackrel{\kappa}{\Phi} \stackrel{\iota}{ }$ represents an entropy flux from the $\kappa$ entity to the $\iota$ entity; and $\Lambda^{\bar{\imath}}$ accounts for entropy production per unit volume due to irreversible processes within the $\iota$ entity. Note that the use of the overbars with superscripts is employed to differentiate among types of macroscale quantities that appear. For example, superscript 
$\iota$ indicates the quantity is an average over the entity of interest (as in eqn (13)); superscript $\bar{\imath}$ indicates the quantity is a mass weighted average over the entity of interest (as in eqn (10)); and the superscript $\overline{\bar{l}}$ designates that the macroscale quantity has been obtained as some other kind of average and/or combination of terms that has been specifically listed in defining the quantity [as in eqns (9), (11), and (12)].

The terms expressing the transfer of a quantity between a phase and an interface can be explained more thoroughly. The macroscale entropy balance equation for an entity in a single-phase flow system has exchange terms of the form

$$
\stackrel{w s \rightarrow \iota}{M_{\eta}}+\stackrel{w s \rightarrow \iota}{\Phi}=\frac{\eta^{\overline{\bar{c}}}}{\epsilon^{\iota} \rho^{\iota}} \stackrel{w s \rightarrow \iota}{M}+\stackrel{w s \rightarrow \iota}{\Phi}=\left\langle\mathbf{n}_{\iota} \cdot\left[\boldsymbol{\varphi}_{\iota}+\eta_{\iota}\left(\mathbf{v}_{w s}-\mathbf{v}_{\iota}\right)\right]\right\rangle_{\Omega_{w s}, \Omega}
$$

where

$$
\begin{gathered}
\stackrel{w \vec{M}^{\iota}}{M}=\left\langle\mathbf{n}_{\iota} \cdot\left[\rho_{\iota}\left(\mathbf{v}_{w s}-\mathbf{v}_{\iota}\right)\right]\right\rangle_{\Omega_{w s}, \Omega} \\
\stackrel{w s \rightarrow \iota}{\Phi}=\left\langle\mathbf{n}_{\iota} \cdot\left[\boldsymbol{\varphi}_{\iota}+\left(\frac{\eta_{\iota}}{\rho_{\iota}}-\frac{\eta^{\bar{\imath}}}{\epsilon^{\iota} \rho^{\iota}}\right) \rho_{\iota}\left(\mathbf{v}_{w s}-\mathbf{v}_{\iota}\right)\right]\right\rangle_{\Omega_{w s}, \Omega}
\end{gathered}
$$

$\iota \in \mathcal{J}_{p}=\{w, s\} ; \mathbf{n}_{\iota}$ is a normal vector pointing outward from phase $\iota$; and $\mathbf{v}_{w s}$ is the microscale velocity of the ws interface.

Also note that we adopt the convention

$$
\begin{aligned}
\stackrel{\iota \rightarrow w s}{M_{\eta}}+\stackrel{\iota \rightarrow w s}{\Phi}=-\stackrel{w s \rightarrow \iota}{M_{\eta}}-\stackrel{w s \rightarrow \iota}{\Phi} & =-\frac{\eta^{\bar{\imath}}}{\epsilon^{\iota} \rho^{\iota}} \stackrel{w s \rightarrow \iota}{M}-\stackrel{w s \rightarrow \iota}{\Phi} \\
& =-\left\langle\mathbf{n}_{\iota} \cdot\left[\boldsymbol{\varphi}_{\iota}+\eta_{\iota}\left(\mathbf{v}_{w s}-\mathbf{v}_{\iota}\right)\right]\right\rangle_{\Omega_{w s}, \Omega}
\end{aligned}
$$

Thus the native definitions for exchange terms involve averaging operators specified in terms of the higher dimensional entity evaluated at the boundary with the lower dimensional entity. This is a common notion that generalizes naturally to the conservation equations and more complex systems. Use of this 
notation for exchange terms along with set notation will allow the formulation of a complete model to be expressed relatively compactly.

Summing the entropy entity balance equation over all entities yields a system entropy inequality equation of the form

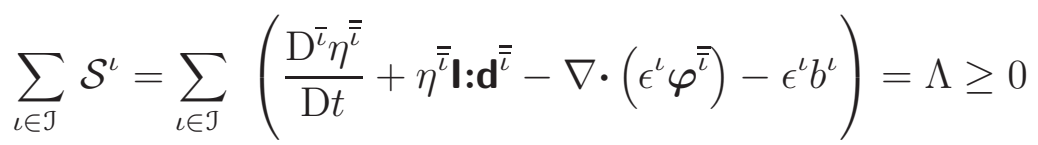

Eqn (20) is the key inequality used to guide the development of thermodynamically constrained closure relations, where the inequality portion of this relation follows from the second law of thermodynamics. Note that when en-

tropy is summed over all entities, the entity exchange terms cancel, giving the simplified expression noted above. To exploit the system entropy inequality relation, we must express entropy as a function of dependent variables appearing in the conservation equations for mass, momentum, and energy.

\section{Conservation Equations}

The set of conservation equations of relevance for the single-phase flow problem includes balances for mass, momentum, and energy for each entity in the index set $\mathcal{J}$. This results in a total of 15 scalar conservations equations. Because our goal is to formulate a model at the macroscale, the macroscale EI will be constrained by the macroscale conservation equations. To be clear, other choices for the type of problem (e.g., multiple-fluid-phase flow, fluid flow and species transport) or the specification of any different scale of concern would influence the final set of conservation equations used to constrain the EI. Other potential choices of scales are detailed in other works in this series [25]. 
After selection of the set of conservation equations needed and the scale of the problem, the conservation equations may be derived at that scale in a variety of ways. If such derivations have already been accomplished for use in other work, the final resultant forms may be used directly. General conservation equations at the required scale can be derived by averaging from the microscale to the macroscale or through a localization approach whereby macroscale equations are written directly in terms of averaged microscale variables. General macroscale conservation equations can then be used to provide specific conservation equations for mass, momentum, and energy. We summarize these macroscale equations without derivation since detailed derivations are available elsewhere [17].

A general equation for mass conservation of the $\iota$ entity is

$$
\mathcal{M}^{\iota}=\frac{\mathrm{D}^{\bar{\imath}}\left(\epsilon^{\iota} \rho^{\iota}\right)}{\mathrm{D} t}+\epsilon^{\iota} \rho^{\iota} \mathbf{I}: \mathbf{d}^{\overline{\bar{\iota}}}-\sum_{\kappa \in \mathcal{J}_{\mathrm{c} \iota}} \stackrel{\kappa \rightarrow}{M} \stackrel{\stackrel{\leftrightarrow}{M}}{ }=0, \quad \text { for } \iota \in \mathcal{J}
$$

where, following our convention for all boundary exchange terms, mass transfer from the $w s$ interface to the $\kappa$ phase is

$$
\stackrel{w s \rightarrow \kappa}{M}=-\stackrel{\kappa \rightarrow m s}{M}=\left\langle\rho_{\kappa} \mathbf{n}_{\kappa} \cdot\left(\mathbf{v}_{w s}-\mathbf{v}_{\kappa}\right)\right\rangle_{\Omega_{w s}, \Omega}, \quad \text { for } \kappa \in \mathcal{J}_{p}
$$

A general equation for momentum conservation of the $\iota$ entity is

$$
\begin{aligned}
\mathcal{P}^{\iota} & =\frac{\mathrm{D}^{\bar{\iota}}\left(\epsilon^{\iota} \rho^{\iota} \mathbf{v}^{\bar{\iota}}\right)}{\mathrm{D} t}+\epsilon^{\iota} \rho^{\iota} \mathbf{v}^{\bar{\iota}} \mathbf{I}: \mathbf{d}^{\bar{\imath}}-\nabla \cdot\left(\epsilon^{\iota} \mathbf{t}^{\bar{\imath}}\right)-\epsilon^{\iota} \rho^{\iota} \mathbf{g}^{\bar{\imath}} \\
& -\sum_{\kappa \in \mathcal{J}_{c \iota}}\left(\stackrel{\kappa \rightarrow \iota}{\mathbf{M}_{v}^{\iota}}+\stackrel{\kappa \rightarrow \iota}{\mathbf{T}}\right)=0, \quad \text { for } \iota \in \mathcal{J}
\end{aligned}
$$

where the macroscale stress tensor is

$$
\mathbf{t}^{\overline{\bar{\iota}}}=\left\langle\mathbf{t}_{\iota}-\rho_{\iota}\left(\mathbf{v}_{\iota}-\mathbf{v}^{\bar{\iota}}\right)\left(\mathbf{v}_{\iota}-\mathbf{v}^{\bar{\iota}}\right)\right\rangle_{\Omega_{\iota}, \Omega_{\iota}}
$$

momentum transfer due to mass exchange from the $w s$ interface to the $\kappa$ phase 
is

$$
\stackrel{w s \rightarrow^{\kappa}}{\mathbf{M}_{v}}=-\stackrel{\kappa \rightarrow w s}{\mathbf{M}_{v}}=\mathbf{v}^{\bar{\kappa}^{w}} \stackrel{w}{M}, \quad \text { for } \kappa \in \mathcal{J}_{p}
$$

and the momentum exchange vector from the $w s$ interface to the $\kappa$ phase due to stress at the boundary is

$$
\stackrel{w s \rightarrow \kappa}{\mathbf{T}}=-\stackrel{\kappa \rightarrow w s}{\mathbf{T}}=\left\langle\mathbf{n}_{\kappa} \cdot\left[\mathbf{t}_{\kappa}+\rho_{\kappa}\left(\mathbf{v}_{w s}-\mathbf{v}_{\kappa}\right)\left(\mathbf{v}_{\kappa}-\mathbf{v}^{\bar{\kappa}}\right)\right]\right\rangle_{\Omega_{w s}, \Omega}, \quad \text { for } \kappa \in \mathcal{J}_{p}
$$

$\mathbf{t}$ is the stress tensor, and $\mathbf{g}$ is the gravitational acceleration vector.

A general equation for total energy conservation of the $\iota$ entity is

$$
\begin{aligned}
& \mathcal{E}^{\iota}=\frac{\mathrm{D}^{\bar{\imath}}\left[E^{\overline{\bar{\iota}}}+\epsilon^{\iota} \rho^{\iota}\left(\frac{1}{2} \mathbf{v}^{\bar{\tau}} \cdot \mathbf{v}^{\bar{\iota}}+K_{E}^{\overline{\bar{\tau}}}+\psi^{\bar{\iota}}\right)\right]}{\mathrm{D} t} \\
& +\left[E^{\overline{\bar{\iota}}}+\epsilon^{\iota} \rho^{\iota}\left(\frac{1}{2} \mathbf{v}^{\bar{\iota}} \cdot \mathbf{v}^{\bar{\iota}}+K_{E}^{\overline{\bar{\iota}}}+\psi^{\bar{\iota}}\right)\right] \mathbf{I}: \mathbf{d}^{\overline{\bar{\tau}}}-\nabla \cdot\left(\epsilon^{\iota} \mathbf{t}^{\overline{\bar{\iota}}} \cdot \mathbf{v}^{\bar{\iota}}+\epsilon^{\iota} \mathbf{q}^{\overline{\bar{\iota}}}\right) \\
& -\epsilon^{\iota} h^{\iota}-\sum_{\kappa \in \mathcal{J}_{\mathrm{c} \iota}}\left(\stackrel{\kappa \rightarrow}{M}_{E}^{\iota}+{\stackrel{\kappa \rightarrow}{T_{v}}}^{\iota}+\stackrel{\kappa \vec{Q}^{\iota}}{\mathrm{Q}}\right)=0, \quad \text { for } \iota \in \mathcal{J}
\end{aligned}
$$

or

$$
\begin{aligned}
\mathcal{E}^{\iota} & =\frac{\mathrm{D}^{\bar{\imath}} E_{T}^{\overline{\bar{\tau}}}}{\mathrm{D} t}+E_{T}^{\overline{\bar{\tau}}} \mathbf{I}: \mathbf{d}^{\overline{\bar{\iota}}}-\nabla \cdot\left(\epsilon^{\iota} \mathbf{t}^{\overline{\bar{\tau}}} \cdot \mathbf{v}^{\bar{\imath}}+\epsilon^{\iota} \mathbf{q}^{\overline{\bar{\tau}}}\right)-\epsilon^{\iota} h^{\iota} \\
& -\sum_{\kappa \in \mathcal{J}_{\mathrm{c} \iota}}\left(\stackrel{\kappa \rightarrow}{M}_{E}^{\iota}+\stackrel{\kappa \rightarrow}{T_{v}^{\iota}}+{ }^{\kappa \rightarrow \iota}{ }^{\iota}\right)=0, \quad \text { for } \iota \in \mathcal{J}
\end{aligned}
$$

where

$$
\begin{aligned}
& E^{\overline{\bar{l}}}=\left\langle E_{\iota}\right\rangle_{\Omega_{\iota}, \Omega} \\
& \epsilon^{\iota} \rho^{\iota} K_{E}^{\overline{\bar{\tau}}}=\left\langle\frac{\rho_{\iota}}{2}\left(\mathbf{v}_{\iota}-\mathbf{v}^{\bar{\tau}}\right) \cdot\left(\mathbf{v}_{\iota}-\mathbf{v}^{\bar{\tau}}\right)\right\rangle_{\Omega_{\iota}, \Omega} \\
& E_{T}^{\overline{\bar{\iota}}}=E^{\overline{\bar{\iota}}}+\epsilon^{\iota} \rho^{\iota}\left(\frac{1}{2} \mathbf{v}^{\bar{\iota}} \cdot \mathbf{v}^{\bar{\iota}}+K_{E}^{\overline{\bar{\iota}}}+\psi^{\bar{\iota}}\right) \\
& \epsilon^{\iota} \mathbf{q}^{\overline{\bar{\iota}}}=\left\langle\mathbf{q}_{\iota}+\left(\frac{E_{\iota}}{\rho_{\iota}}-\frac{E^{\overline{\bar{\iota}}}}{\epsilon^{\iota} \rho^{\iota}}+\frac{1}{2}\left(\mathbf{v}_{\iota}-\mathbf{v}^{\bar{\iota}}\right) \cdot\left(\mathbf{v}_{\iota}-\mathbf{v}^{\bar{\tau}}\right)\right) \rho_{\iota}\left(\mathbf{v}_{\iota}-\mathbf{v}^{\bar{\tau}}\right)\right\rangle_{\Omega_{\iota}, \Omega} \\
& +\left\langle\left(-K_{E}^{\overline{\bar{\tau}}}+\psi_{\iota}-\psi^{\bar{\tau}}\right) \rho_{\iota}\left(\mathbf{v}_{\iota}-\mathbf{v}^{\bar{\tau}}\right)+\mathbf{t}_{\iota} \cdot\left(\mathbf{v}_{\iota}-\mathbf{v}^{\bar{\tau}}\right)\right\rangle_{\Omega_{\iota}, \Omega} \\
& \stackrel{w s \rightarrow \kappa}{M_{E}}=-\stackrel{\kappa \rightarrow w s}{M}_{E}=\frac{E_{T}^{\overline{\bar{\kappa}}}}{\epsilon^{\kappa} \rho^{\kappa}} \stackrel{w \rightarrow \kappa}{M}, \quad \text { for } \kappa \in \mathcal{J}_{p}
\end{aligned}
$$




$$
\begin{aligned}
& \stackrel{w s \rightarrow \kappa}{T_{v}}=-{ }^{\kappa \rightarrow} T_{v}^{w s}=\stackrel{w s \rightarrow \kappa}{\mathbf{T}} \cdot \mathbf{v}^{\bar{\kappa}}, \quad \text { for } \kappa \in \mathcal{J}_{p} \\
& \stackrel{w s \rightarrow \kappa}{Q}=-\stackrel{\kappa \rightarrow}{Q} \stackrel{w s}{Q}=\left\langle\mathbf{n}_{\kappa} \cdot \mathbf{q}_{\kappa}+\mathbf{n}_{\kappa} \cdot \mathbf{t}_{\kappa} \cdot\left(\mathbf{v}_{\kappa}-\mathbf{v}^{\bar{\kappa}}\right)\right\rangle_{\Omega_{w s}, \Omega} \\
& +\left\langle\left(\frac{E_{\kappa}}{\rho_{\kappa}}-\frac{E^{\overline{\bar{\kappa}}}}{\epsilon^{\kappa} \rho^{\kappa}}\right) \rho_{\kappa}\left(\mathbf{v}_{w s}-\mathbf{v}_{\kappa}\right) \cdot \mathbf{n}_{\kappa}\right\rangle_{\Omega_{w s}, \Omega} \\
& +\left\langle\frac{1}{2}\left(\mathbf{v}_{\kappa}-\mathbf{v}^{\bar{\kappa}}\right) \cdot\left(\mathbf{v}_{\kappa}-\mathbf{v}^{\bar{\kappa}}\right) \rho_{\kappa}\left(\mathbf{v}_{w s}-\mathbf{v}_{\kappa}\right) \cdot \mathbf{n}_{\kappa}\right\rangle_{\Omega_{w s}, \Omega} \\
& +\left\langle\left(\psi_{\kappa}-\psi^{\bar{\kappa}}-K_{E}^{\overline{\bar{\kappa}}}\right) \rho_{\kappa}\left(\mathbf{v}_{w s}-\mathbf{v}_{\kappa}\right) \cdot \mathbf{n}_{\kappa}\right\rangle_{\Omega_{w s}, \Omega},
\end{aligned}
$$

and $E_{T}^{\overline{\bar{\tau}}}$ is the total energy; $K_{E}^{\overline{\bar{\tau}}}$ is the kinetic energy due to microscale velocity fluctuations; $\psi^{\bar{\tau}}$ is the gravitational potential; $\mathbf{q}^{\overline{\bar{l}}}$ is the heat flux vector; $h^{\iota}$ is a heat source, $\stackrel{w s \rightarrow \kappa}{M_{E},}, \stackrel{w s \rightarrow \kappa}{T_{v}}$, and $\stackrel{w s \rightarrow \kappa}{Q}$ express the transfer of energy from the ws interface to the $\kappa$ phase due to interfacial stress, phase change, and heat transfer. The $K_{E}^{\overline{\bar{\tau}}}$ terms have traditionally been neglected or lumped in with other macroscale quantities, such as the internal energy. When they contribute negligibly to the energy of the porous media system, as is typically the case, such an approach is acceptable. For completeness, we carry these terms explicitly.

Thus we have three conservation equations for mass, nine for momentum, and three for energy, making a total of 15 conservation equations. These equations are not solvable as written, because the set of unknown variables includes 64 unknown quantities

$$
\mathcal{V}=\left\{\epsilon^{\iota}, \rho^{\iota}, \mathbf{v}^{\bar{\iota}}, \stackrel{w s \rightarrow \kappa}{M}, \mathbf{t}^{\overline{\bar{\iota}}}, \stackrel{w s \rightarrow \kappa}{\mathbf{T}}, E^{\overline{\bar{\iota}}}, K_{E}^{\overline{\bar{\tau}}}, \psi^{\bar{\tau}}, \mathbf{q}^{\overline{\bar{\iota}}}, h^{\iota}, \stackrel{w s \rightarrow \kappa}{Q}\right\}
$$

for $\iota \in \mathcal{J}$ and $\kappa \in \mathcal{J}_{p}$ and with $\mathbf{t}^{\bar{\tau}}$ symmetric. Specification of single-phase models therefore requires some combination of simplifying assumptions and additional relations such that for a particular problem of interest the number of unknown quantities is equivalent to the number of equations. We refer to 
finding conditions that make the number of unknowns equal to the number of equations as the closure problem, and the specification of thermodynamically constrained and closed models is a focus of this work.

\section{Thermodynamics}

Three aspects of thermodynamics are important in the development of TCAT

models: (1) averaged macroscale expressions that relate material derivatives of internal energy to material derivatives of entropy, mass, and geometric measures for near equilibrium conditions; (2) a set of macroscale equalities that must hold at equilibrium; and (3) considerations involving the production, flux, and source of entropy. We summarize these three classes of macroscale thermodynamic results in turn below.

\subsection{Material Derivatives of Internal Energy}

Eqn (20) contains terms accounting for entropy, non-advective entropy fluxes, and entropy source terms that do not appear in any of the conservation equations used to produce an AEI. To link the entropy to other terms appearing in the AEI, we rely upon macroscale thermodynamics to provide relations between entropy and other quantities appearing in conservation equations.

As previously discussed [20], multiple approaches exist for positing the thermodynamics needed to provide the necessary link between entropy and the set of conserved quantities. Further, issues of scale arise in developing these thermodynamic expressions. In this work, we collect previously derived results based upon averaging a classical irreversible thermodynamic (CIT) approach 
at the microscale to the macroscale. CIT is based upon an assumption that a system may be subdivided into discrete spatial and temporal regions with equilibrium thermodynamics applied locally in those regions. This view of system behavior is termed the local equilibrium assumption, and it is a distinguishing feature of CIT compared to classical equilibrium thermodynamics [20]. CIT is thus a convenient theory upon which to build porous medium models, but it is not the only, or perhaps even the best, choice available. We utilize results from this approach here because it is a reasonable starting point in our quest to develop consistent, TCAT-based models that are closed and specific.

Because of differences in the thermodynamic functional forms among the entities, we consider each entity in turn. The forms desired are expressions involving the material derivatives of internal energy for each entity in the system that equate to zero. Based on Gray [16], the material derivative form involving $E^{\overline{\bar{w}}}$ is

$$
\begin{aligned}
\mathcal{T}^{w} & =\frac{\mathrm{D}^{\bar{w}} E^{\overline{\bar{w}}}}{\mathrm{D} t}-\theta^{\overline{\bar{w}}} \frac{\mathrm{D}^{\bar{w}} \eta^{\overline{\bar{w}}}}{\mathrm{D} t}-\mu^{\bar{w}} \frac{\mathrm{D}^{\bar{w}}\left(\epsilon^{w} \rho^{w}\right)}{\mathrm{D} t}+p^{w} \frac{\mathrm{D}^{\bar{w}} \epsilon^{w}}{\mathrm{D} t} \\
& +\left\langle\eta_{w} \frac{\mathrm{D}^{\bar{w}}\left(\theta_{w}-\theta^{\overline{\bar{w}}}\right)}{\mathrm{D} t}+\rho_{w} \frac{\mathrm{D}^{\bar{w}}\left(\mu_{w}-\mu^{\bar{w}}\right)}{\mathrm{D} t}-\frac{\mathrm{D}^{\bar{w}}\left(p_{w}-p^{w}\right)}{\mathrm{D} t}\right\rangle_{\Omega_{w}, \Omega}=0
\end{aligned}
$$

where $\theta^{\overline{\bar{w}}}$ is the temperature, $p^{w}$ is the fluid pressure, and $\mu^{\bar{w}}$ is the chemical potential. Note that each of the time derivatives of a difference between a macroscale quantity and its microscale precursor is contained in an integral over $\Omega_{w}$. Thus if the system is spatially homogeneous at the microscale in one of these properties, the corresponding integral term will vanish. Even if the system is not microscopically spatially homogeneous, the integral terms involving the time derivatives may vanish. In general however, these integral terms are not zero, but in many practical cases they may be small. The presence of these extra terms represents a difference between formulations based 
upon averaged microscale thermodynamics and formulations based upon thermodynamics posited directly at the macroscale.

Averaging the CIT from the microscale to the macroscale for a solid phase gives [21]

$$
\begin{aligned}
\mathcal{T}^{s} & =\frac{\mathrm{D}^{\bar{s}} E^{\overline{\bar{s}}}}{\mathrm{D} t}-\theta^{\overline{\bar{s}}} \frac{\mathrm{D}^{\bar{s}} \eta^{\overline{\bar{s}}}}{\mathrm{D} t}-\mu^{\bar{s}} \frac{\mathrm{D}^{\bar{s}}\left(\epsilon^{s} \rho^{s}\right)}{\mathrm{D} t}+\left\langle\eta_{s} \frac{\mathrm{D}^{\bar{s}}\left(\theta_{s}-\theta^{\overline{\bar{s}}}\right)}{\mathrm{D} t}+\rho_{s} \frac{\mathrm{D}^{\bar{s}}\left(\mu_{s}-\mu^{\bar{s}}\right)}{\mathrm{D} t}\right\rangle_{\Omega_{s}, \Omega} \\
& -\left\langle\left(\frac{\mathbf{C}_{s}}{j_{s}}: \boldsymbol{\sigma}_{s}\right)\left(\mathbf{v}_{w s}-\mathbf{v}_{s}\right) \cdot \mathbf{n}_{s}\right\rangle_{\Omega_{w s}, \Omega} \\
& -\left\langle\mathbf{n}_{s} \cdot\left[\frac{2}{j_{s}} \boldsymbol{\sigma}_{s}:\left(\nabla_{X} \mathbf{x} \nabla_{X} \mathbf{x}\right) \cdot\left(\mathbf{v}_{s}-\mathbf{v}^{\bar{s}}\right)\right]\right\rangle_{\Omega_{w s}, \Omega} \\
& +\left\langle\left\{\nabla \cdot\left[\frac{2}{j_{s}} \boldsymbol{\sigma}_{s}:\left(\nabla_{X} \mathbf{x} \nabla_{X} \mathbf{x}\right)\right]-\nabla_{s}: \frac{\mathbf{C}_{s}}{j_{s}}\right\} \cdot\left(\mathbf{v}_{s}-\mathbf{v}^{\bar{s}}\right)\right\rangle_{\Omega_{s}, \Omega} \\
& +\epsilon^{s} \boldsymbol{\sigma}^{s}: \frac{\mathbf{C}^{s}}{j^{s}} \mathbf{I}: \mathbf{d}^{\bar{s}}-\left\langle\frac{2}{j_{s}} \boldsymbol{\sigma}_{s}:\left(\nabla_{X} \mathbf{x} \nabla_{X} \mathbf{x}\right)\right\rangle_{\Omega_{s}, \Omega}: \mathbf{d}^{\bar{s}} \\
& -\nabla \cdot\left\langle\left[\frac{2}{j_{s}} \boldsymbol{\sigma}_{s}:\left(\nabla_{X} \mathbf{x} \nabla_{X} \mathbf{x}\right)-\boldsymbol{\sigma}_{s}: \frac{\mathbf{C}_{s}}{j_{s}} \mathbf{l}\right] \cdot\left(\mathbf{v}_{s}-\mathbf{v}^{\bar{s}}\right)\right\rangle_{\Omega_{s}, \Omega}=0
\end{aligned}
$$

where $\boldsymbol{\sigma}_{s}$ is the Lagrangian stress tensor, $\mathbf{C}_{s}$ is the Greens' deformation tensor, $j_{s}=\left|\partial x_{i} / \partial X_{j}\right|$ is the solid-phase Jacobian, $\mathbf{x}$ represents the position in the solid phase, $\mathbf{X}$ represents the initial position in the solid phase, $\nabla_{X} \mathbf{X}$ is the gradient of a spatial location vector relative to its initial location, and subscripts denote microscale quantities while superscripts denote macroscale quantities.

Averaging the CIT expression for the ws interface from the microscale to the macroscale gives [16]

$$
\begin{aligned}
\mathcal{T}^{w s} & =\frac{\mathrm{D}^{\overline{w s}} E^{\overline{\overline{w s}}}}{\mathrm{D} t}-\theta^{\overline{\overline{w s}}} \frac{\mathrm{D}^{\overline{w s}} \eta^{\overline{\overline{w s}}}}{\mathrm{D} t}-\mu^{\overline{w s}} \frac{\mathrm{D}^{\overline{w s}}\left(\epsilon^{w s} \rho^{w s}\right)}{\mathrm{D} t}-\gamma^{w s} \frac{\mathrm{D}^{\overline{w s}} \epsilon^{w s}}{\mathrm{D} t} \\
& +\left\langle\eta_{w s} \frac{\mathrm{D}^{\overline{w s}}\left(\theta_{w s}-\theta^{\overline{\overline{w s}}}\right)}{\mathrm{D} t}+\rho_{w s} \frac{\mathrm{D}^{\overline{w s}}\left(\mu_{w s}-\mu^{\overline{w s}}\right)}{\mathrm{D} t}\right\rangle_{\Omega_{w s}, \Omega} \\
& +\left\langle\frac{\mathrm{D}^{\overline{w s}}\left(\gamma_{w s}-\gamma^{w s}\right)}{\mathrm{D} t}\right\rangle_{\Omega_{w s}, \Omega}-\nabla \theta^{\overline{\overline{w s}}} \cdot\left\langle\mathbf{n}_{w} \mathbf{n}_{w} \cdot\left(\mathbf{v}^{\overline{w s}}-\mathbf{v}_{w s}\right) \eta_{w s}\right\rangle_{\Omega_{w s}, \Omega}
\end{aligned}
$$




$$
\begin{aligned}
& -\nabla \mu^{\overline{w s}} \cdot\left\langle\mathbf{n}_{w} \mathbf{n}_{w} \cdot\left(\mathbf{v}^{\overline{w s}}-\mathbf{v}_{w s}\right) \rho_{w s}\right\rangle_{\Omega_{w s}, \Omega} \\
& -\nabla \gamma^{w s} \cdot\left\langle\mathbf{n}_{w} \mathbf{n}_{w} \cdot\left(\mathbf{v}^{\overline{w s}}-\mathbf{v}_{w s}\right)\right\rangle_{\Omega_{w s}, \Omega}
\end{aligned}
$$

for

$$
\begin{gathered}
\frac{\mathrm{D}^{\overline{w s}}}{\mathrm{D} t}=\frac{\partial^{\prime}}{\partial t}+\mathbf{v}^{\overline{w s}} \cdot \nabla^{\prime} \\
\frac{\partial^{\prime}}{\partial t}=\frac{\partial}{\partial t}+\mathbf{v}_{w s} \cdot \mathbf{n}_{w} \mathbf{n}_{w} \cdot \nabla \\
\nabla^{\prime}=\nabla-\mathbf{n}_{w} \mathbf{n}_{w} \cdot \nabla
\end{gathered}
$$

and

$$
\frac{\mathrm{D}^{\overline{w s}}}{\mathrm{D} t}=\frac{\mathrm{D}^{\overline{w s}}}{\mathrm{D} t}+\left(\mathbf{v}_{w s}-\mathbf{v}^{\overline{w s}}\right) \cdot \mathbf{n}_{w} \mathbf{n}_{w} \cdot \nabla
$$

where $\gamma$ is the interfacial tension. The thermodynamics of interfaces at the macroscale require restriction of microscale quantities to the interface and averaging of these quantities to the macroscale. Because of this, the material derivatives within the averaging operators are restricted to the interface at the microscale.

These equations provide explicit links between entropy and other macroscale variables that appear in the conservation equations that we wish to close. These links are essential to the formulation of closed models.

\subsection{Equilibrium Conditions}

In order to exploit the system EI to guide the formulation of appropriate closure relations, it is beneficial to arrange the EI into products of independent variables and groupings of terms known to vanish at equilibrium. The development of such equilibrium conditions for microscale properties can be accomplished using variational methods $[1,6,7]$. Then, averaging of the microscale equilibrium conditions provides the macroscale constraints. We will 
not detail these methods in this work, rather we will summarize results that will be of use in guiding manipulations of the EI, which were derived using variational methods [21]. At equilibrium, all velocities are constant and equal such that

$$
\mathbf{v}^{\bar{w}}=\mathbf{v}^{\bar{s}}=\mathbf{v}^{\overline{w s}}=\text { constant }
$$

and as a consequence of this condition

$$
\mathbf{d}^{\overline{\bar{w}}}=\mathbf{d}^{\overline{\bar{s}}}=\mathbf{d}^{\overline{\overline{w s}}}=0
$$

The macroscale temperatures are also constant and equal for all entities with

$$
\theta^{\overline{\bar{w}}}=\theta^{\overline{\bar{s}}}=\theta^{\overline{\overline{w s}}}=\text { constant }
$$

and as a consequence of this condition

$$
\nabla \theta^{\overline{\bar{w}}}=\nabla \theta^{\overline{\bar{s}}}=\nabla \theta^{\overline{\overline{w s}}}=0
$$

At equilibrium, the sum of chemical and gravitational potentials are related as follows

$$
\begin{aligned}
& \mu^{\bar{w}}+\psi^{\bar{w}}=\mu^{\overline{w s}}+\psi^{\overline{w s}}= \\
& \mu^{\bar{s}}+\psi^{\bar{s}}+\left\langle\boldsymbol{\sigma}_{s}: \frac{\mathbf{C}_{s}}{\rho_{s} j_{s}}\right\rangle_{\Omega_{s}, \Omega_{s}}-\frac{1}{\rho^{s}}\left\langle\frac{\mathbf{t}_{s}: \mathbf{I}}{3}\right\rangle_{\Omega_{s}, \Omega_{s}}=\text { constant }
\end{aligned}
$$

and as a consequence of this condition

$$
\begin{aligned}
& \nabla\left(\mu^{\bar{w}}+\psi^{\bar{w}}\right)=\nabla\left(\mu^{\bar{s}}+\psi^{\bar{s}}+\left\langle\boldsymbol{\sigma}_{s}: \frac{\mathbf{C}_{s}}{\rho_{s} j_{s}}\right\rangle_{\Omega_{s}, \Omega_{s}}-\frac{1}{\rho^{s}}\left\langle\frac{\mathbf{t}_{s}: \mathbf{I}}{3}\right\rangle_{\Omega_{s}, \Omega_{s}}\right)= \\
& \nabla\left(\mu^{\overline{w s}}+\psi^{\overline{w s}}\right)=0
\end{aligned}
$$

where

$$
\mathbf{t}_{s}=\frac{2}{j_{s}} \boldsymbol{\sigma}_{s}:\left(\nabla_{X} \mathbf{x} \nabla_{X} \mathbf{x}\right)
$$


The macroscale geometric variables are constant at equilibrium so

$$
\frac{\mathrm{D}^{\bar{w}} \epsilon^{w}}{\mathrm{D} t}=\frac{\mathrm{D}^{\bar{s}} \epsilon^{s}}{\mathrm{D} t}=\frac{\mathrm{D}^{\overline{w s}} \epsilon^{w s}}{\mathrm{D} t}=0
$$

Within the solid phase the equilibrium condition is

$$
\left\langle\rho_{s} \nabla\left(\frac{\mathbf{t}_{s}: \mathbf{I}}{3 \rho_{s}}\right)-\nabla \cdot \mathbf{t}_{s}\right\rangle_{\Omega_{s}, \Omega_{s}}=0
$$

Eqn (48) and eqn (52) can be used along with the Gibbs-Duhem equation to deduce the equilibrium condition

$$
\left\langle\nabla \cdot \mathbf{t}_{s}-\nabla \boldsymbol{\sigma}_{s}: \frac{\mathbf{C}_{s}}{j_{s}}\right\rangle_{\Omega_{s}, \Omega_{s}}=0
$$

The expression for the balance of normal stress at the fluid-solid interface is

$$
\left\langle p_{w}+\mathbf{n}_{s} \cdot \mathbf{t}_{s} \cdot \mathbf{n}_{s}+\gamma_{w s} \nabla^{\prime} \cdot \mathbf{n}_{s}-\rho_{w s} \mathbf{g}_{w s} \cdot \mathbf{n}_{s}\right\rangle_{\Omega_{w s}, \Omega_{w s}}=0
$$

while the lateral stress at the solid surface obeys the equilibrium condition

$$
\left\langle\mathbf{n}_{s} \cdot \mathbf{t}_{s} \cdot \mathbf{I}^{\prime}\right\rangle_{\Omega_{w s}, \Omega_{w s}}=0
$$

where $\mathbf{I}^{\prime}=\mathbf{I}-\mathbf{n}_{s} \mathbf{n}_{s}$ is the surface identity tensor.

\subsection{Production, Flux, and Source of Entropy}

Within the CIT framework, entropy production is a result of heat conduction, the flow of matter, mechanical dissipation, chemical reactions, and electrical currents leading to irreversible processes [23]. It is a standard procedure to seek a form that represents these entropy producing processes as the product 
of a set of thermodynamic fluxes and thermodynamic forces such that

$$
\Lambda=\sum_{i} J_{i} F_{i}+\sum_{j} \mathbf{J}_{j} \cdot \mathbf{F}_{j}+\sum_{k} \mathbf{J}_{k}: \mathbf{F}_{k}
$$

where $J_{i}, \mathbf{J}_{j}$, and $\mathbf{J}_{k}$ are thermodynamic fluxes corresponding to scalar, vector, and tensor types, respectively; and $F_{i}, \mathbf{F}_{j}$, and $\mathbf{F}_{k}$ are thermodynamic forces of the scalar, vector, and tensor types, respectively.

An important property of these products is that each factor in all products must be zero at equilibrium - the state of minimum energy, maximum entropy, and no entropy production. Another property is that each member of the set of fluxes is independent of all other fluxes, and each member of the set of forces is independent of all other forces, which collectively we will refer to as the flux-force independence condition. Because of the symmetry required by the flux-force independence condition, Jou et al. [23] comment that the identification of each factor in the products given in eqn (56) as a "force" or "flux" is arbitrary as long as the independence condition is met.

Although the thermodynamic flux-force approach is common in the CIT literature for microscale systems, care is needed when extending these notions to the macroscale systems of concern in this work. We are not aware of any available approach to generate a complete list of the set of fluxes and forces a priori. We do have two sources of information to provide some guidance: (1) precedent and knowledge based upon microscale systems, and (2) the equilibrium conditions summarized in $\S 5.2$. We will use both of these sources to propose a set of forces that is consistent with eqns (44)-(51) that is also constrained by the independence condition. The conjugate set of fluxes are unknown a priori, but it is known that once deduced these fluxes must be zero at equilibrium and meet the independence condition. These notions are consistent with Postulate 
Postulate 1 (Entropy Production Postulate, EPP) The production of entropy, $\Lambda$, may be expressed as a sum of inner products of members of a set of fluxes $\mathcal{J}$ and conjugate members of a companion set of forces $\mathcal{F}$ with both $\mathcal{I}$ and $\mathcal{F}$ comprised of members that are zero at equilibrium and independent of all other members in the respective set but which can depend upon one or more members of the companion set.

The EPP is valuable because it provides guidance for the development of a form of the EI that will be of most use in developing closure relations, and it leads to linearized approximations for a set of fluxes in terms of members of a set of mutually independent forces in the near-equilibrium regime. It is also important to understand that although the forces are typically clearly zero at equilibrium (e.g., a rate of strain), we are only able to infer that the fluxes are zero on the basis of the EPP, in most cases. In all cases however, confirmation that the fluxes vanish at equilibrium will be verifiable based upon the derived equilibrium conditions and microscale analysis. Therefore, it is important that the forces that we identify as "independent" indeed be independent. We are guided in this pursuit by insight into the system summarized by the equilibrium conditions given in $§ 5.2$.

\section{$6 \quad$ Augmented Entropy Inequality}

In the TCAT approach, the system EI is augmented with the conservation equations and thermodynamic equalities that express the relations between the material derivatives of macroscale internal energy of system entities and 
expressions that appear in the macroscale EI and conservation equations. This augmented EI (AEI) can be interpreted as a statement of the second-law of thermodynamics constrained to ensure that it satisfies the conservation equations and thermodynamic identities evolving from the chosen thermodynamic theory. The AEI may be written as

$$
\sum_{\iota \in \mathcal{J}}\left(\mathcal{S}^{\iota}+\lambda_{\mathcal{M}}^{\iota} \mathcal{M}^{\iota}+\lambda_{\mathcal{P}}^{\iota} \cdot \mathcal{P}^{\iota}+\lambda_{\mathcal{E}}^{\iota} \mathcal{E}^{\iota}+\lambda_{\mathcal{T}}^{\iota} \mathcal{T}^{\iota}\right)=\Lambda \geq 0
$$

where the sub- and superscripted $\lambda$ 's are Lagrange multipliers of the corresponding conservation equations $\mathcal{S}^{\iota}, \mathcal{M}^{\iota}, \mathcal{P}^{\iota}$, and $\mathcal{E}^{\iota}$ given in eqns (20), (21), (23), and (27), respectively, and the thermodynamic expressions $\mathcal{T}^{\iota}$ given in eqns (37)-(39).

In subsequent manipulations to solve for the Lagrange multipliers, our attention will be focused on the material derivatives. We will therefore introduce a shorthand notation for all the terms in the conservation and thermodynamic equations that do not contain material derivatives. Thus entropy eqn (20) is expressed

$$
\sum_{\iota \in \mathcal{J}} \mathcal{S}^{\iota}=\sum_{\iota \in \mathcal{J}}\left(\frac{\mathrm{D}^{\bar{\imath}} \eta^{\bar{\imath}}}{\mathrm{D} t}+\mathcal{S}_{D}^{\iota}\right)=\Lambda \geq 0
$$

Mass conservation eqn (21) is written

$$
\mathcal{M}^{\iota}=\frac{\mathrm{D}^{\bar{\iota}}\left(\epsilon^{\iota} \rho^{\iota}\right)}{\mathrm{D} t}+\mathcal{M}_{D}^{\iota}=0, \quad \text { for } \iota \in \mathcal{J}
$$

Conservation of momentum as expressed by eqn (23) is

$$
\mathcal{P}^{\iota}=\frac{\mathrm{D}^{\bar{\imath}}\left(\epsilon^{\iota} \rho^{\iota} \mathbf{v}^{\bar{\iota}}\right)}{\mathrm{D} t}+\mathcal{P}_{D}^{\iota}=0, \quad \text { for } \iota \in \mathcal{J}
$$

The material derivatives in energy conservation eqn (27) are expanded out using the product rule so that this equation becomes

$$
\mathcal{E}^{\iota}=\frac{\mathrm{D}^{\bar{\iota}} E^{\overline{\bar{\iota}}}}{\mathrm{D} t}+\mathbf{v}^{\bar{\iota}} \cdot \frac{\mathrm{D}^{\bar{\iota}}\left(\epsilon^{\iota} \rho^{\iota} \mathbf{v}^{\bar{\iota}}\right)}{\mathrm{D} t}+\left(K_{E}^{\overline{\bar{\tau}}}-\frac{\mathbf{v}^{\bar{\tau}} \cdot \mathbf{v}^{\bar{\iota}}}{2}+\psi^{\bar{\iota}}\right) \frac{\mathrm{D}^{\bar{\iota}}\left(\epsilon^{\iota} \rho^{\iota}\right)}{\mathrm{D} t}
$$




$$
+\epsilon^{\iota} \rho^{\iota} \frac{\mathrm{D}^{\bar{\iota}}\left(K_{E}^{\overline{\bar{L}}}+\psi^{\bar{\iota}}\right)}{\mathrm{D} t}+\mathcal{E}_{D}^{\iota}=0, \quad \text { for } \iota \in \mathcal{J}
$$

The thermodynamic forms of eqns (37)-(39) for the $w, s$, and $w s$ entities are written, respectively, as

$$
\begin{gathered}
\mathcal{T}^{w}=\frac{\mathrm{D}^{\bar{w}} E^{\overline{\bar{w}}}}{\mathrm{D} t}-\theta^{\overline{\bar{w}}} \frac{\mathrm{D}^{\bar{w}} \eta^{\overline{\bar{w}}}}{\mathrm{D} t}-\mu^{\bar{w}} \frac{\mathrm{D}^{\bar{w}}\left(\epsilon^{w} \rho^{w}\right)}{\mathrm{D} t}+p^{w} \frac{\mathrm{D}^{\bar{w}} \epsilon^{w}}{\mathrm{D} t}+\mathcal{T}_{r}^{w}=0 \\
\mathcal{T}^{s}=\frac{\mathrm{D}^{\bar{s}} E^{\overline{\bar{s}}}}{\mathrm{D} t}-\theta^{\overline{\bar{s}}} \frac{\mathrm{D}^{\bar{s}} \eta^{\overline{\bar{s}}}}{\mathrm{D} t}-\mu^{\bar{s}} \frac{\mathrm{D}^{\bar{s}}\left(\epsilon^{s} \rho^{s}\right)}{\mathrm{D} t}+\mathcal{T}_{r}^{s}=0
\end{gathered}
$$

and

$$
\mathcal{T}^{w s}=\frac{\mathrm{D}^{\overline{w s}} E^{\overline{\overline{w s}}}}{\mathrm{D} t}-\theta^{\overline{\overline{w s}}} \frac{\mathrm{D}^{\overline{w s}} \eta^{\overline{\overline{w s}}}}{\mathrm{D} t}-\mu^{\overline{w s}} \frac{\mathrm{D}^{\overline{w s}}\left(\epsilon^{w s} \rho^{w s}\right)}{\mathrm{D} t}-\gamma^{w s} \frac{\mathrm{D}^{\overline{w s}} \epsilon^{w s}}{\mathrm{D} t}+\mathcal{T}_{r}^{w s}=0
$$

The introduction of notation to account for collections of terms not involving material derivatives is a convenience for use in the next section to facilitate selection of the Lagrange multipliers strictly on the basis of the material derivatives and the physical processes to be modeled.

\section{Constrained Entropy Inequality}

Expanding upon previously introduced Lagrange multiplier approaches for

constraining the entropy inequality [e.g. 20,22], we show explicitly the limits of the simplifications to material derivative expressions possible with any choice of Lagrange multipliers. After the conservation and thermodynamic equations are substituted into eqn (57), a total of 23 distinct material derivatives exist in this inequality, including three components for each entity-momentum term. Additionally, we note that the equation contains 18 scalar Lagrange multipliers. Correspondingly, the vector of all Lagrange multipliers $\boldsymbol{\lambda}$ is of dimension 
18. Examination of eqn (57) in light of basic considerations of solvability reveals that material derivatives of kinetic energy plus gravitational potential and of entity measures for the $w$ and $w s$ entities cannot be eliminated for any set of non-zero Lagrange multipliers. Elimination of these five material derivatives from consideration yields a linear system of 18 equations in 18 unknown Lagrange multipliers, which can be written as

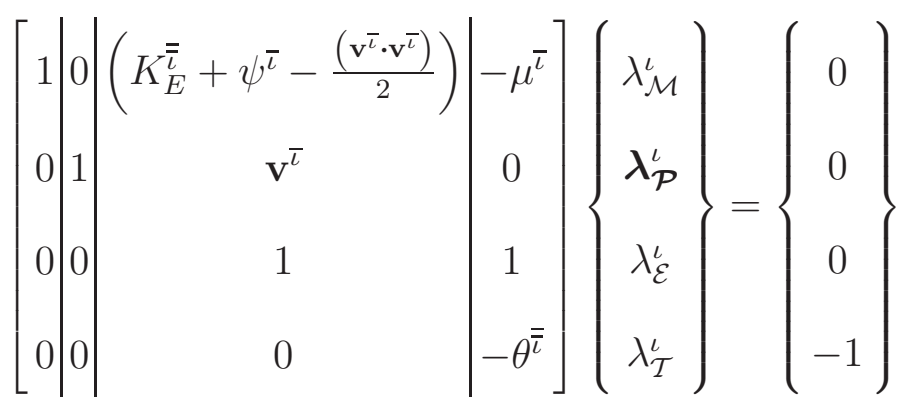

where the rows of the matrix are formed, in order, by collecting coefficients of the material derivatives of mass density, momentum density, internal energy density, and entropy. We have compressed the three momentum terms and the set of entities for each quantity to a single line, noting that these equations are of a similar form.

In light of the upper triangular form of the coefficient matrix in eqn (65), this equation may be readily solved for the Lagrange multipliers to obtain

$$
\left\{\begin{array}{c}
\lambda_{\mathcal{M}}^{\iota} \\
\lambda_{\mathcal{P}}^{\iota} \\
\lambda_{\mathcal{E}}^{\iota} \\
\lambda_{\mathcal{T}}^{\iota}
\end{array}\right\}=\frac{1}{\theta^{\iota}}\left\{\begin{array}{c}
\left(K_{E}^{\overline{\bar{\tau}}}+\mu^{\bar{\tau}}+\psi^{\bar{\iota}}-\frac{\left(\mathbf{v}^{\bar{\tau}} \cdot \mathbf{v}^{\bar{\tau}}\right)}{2}\right) \\
\mathbf{v}^{\bar{\iota}} \\
-1 \\
1
\end{array}\right\}, \quad \text { for } \iota \in \mathcal{J}
$$

Substitution of these results into eqn (57), yields a constrained entropy inequality (CEI) of the form 


$$
\begin{aligned}
\sum_{\iota \in \mathcal{J}} & {\left[\mathcal{S}_{D}^{\iota}+\frac{1}{\theta^{\overline{\bar{\iota}}}}\left(K_{E}^{\overline{\bar{l}}}+\mu^{\bar{\iota}}+\psi^{\bar{\iota}}-\frac{\left(\mathbf{v}^{\bar{\iota}} \cdot \mathbf{v}^{\bar{\iota}}\right)}{2}\right) \mathcal{M}_{D}^{\iota}\right] } \\
& +\sum_{\iota \in \mathcal{J}}\left[\frac{\mathbf{v}^{\bar{\iota}}}{\theta^{\bar{\iota}}} \cdot \mathcal{P}_{D}^{\iota}-\frac{1}{\theta^{\bar{\iota}}}\left(\epsilon^{\iota} \rho^{\iota} \frac{\mathrm{D}^{\bar{\iota}}\left(K_{E}^{\overline{\bar{\iota}}}+\psi^{\bar{\iota}}\right)}{\mathrm{D} t}+\mathcal{E}_{D}^{\iota}\right)\right] \\
& +\frac{1}{\theta^{\overline{\bar{w}}}}\left(p^{w} \frac{\mathrm{D}^{\bar{w}} \epsilon^{w}}{\mathrm{D} t}+\mathcal{T}_{r}^{w}\right)+\frac{\mathcal{T}_{r}^{s}}{\theta^{\overline{\bar{s}}}}-\frac{1}{\theta^{\overline{\overline{w s}}}}\left(\gamma^{w s} \frac{\mathrm{D}^{\overline{w s}} \epsilon^{w s}}{\mathrm{D} t}-\mathcal{T}_{r}^{w s}\right)=\Lambda \geq 0
\end{aligned}
$$

Five macroscale material derivatives remain in this expression as well as several material derivatives within averaging operators associated with the expressions for the averaged thermodynamics. These remaining material derivatives and the velocities are all referenced to a common frame to lead to a form that will satisfy the continuum mechanical axiom of objectivity. We select the macroscale, mass-averaged solid-phase velocity, $\mathbf{v}^{\bar{s}}$, as the reference velocity. Stating the equation in terms of objective quantities, applying averaging theorems [18, 25], and algebraic rearrangement to group in flux-force pairs as described in $§ 5.3$ yields

$$
\begin{aligned}
& -\nabla \cdot\left[\epsilon^{w}\left(\varphi^{\overline{\bar{w}}}-\frac{\mathbf{q}^{\overline{\bar{w}}}}{\theta^{\overline{\bar{w}}}}\right)\right]-\nabla \cdot\left[\epsilon^{w s}\left(\varphi^{\overline{\overline{w s}}}-\frac{\mathbf{q}^{\overline{\overline{w s}}}}{\theta^{\overline{\overline{w s}}}}\right)\right] \\
& -\nabla \cdot\left\{\epsilon^{s} \boldsymbol{\varphi}^{\overline{\bar{s}}}-\frac{1}{\theta^{\overline{\bar{s}}}}\left[\epsilon^{s} \mathbf{q}^{\overline{\bar{s}}}-\left\langle\left(\mathbf{t}_{s}-\boldsymbol{\sigma}_{s}: \frac{\mathbf{C}_{s}}{j_{s}} \mathbf{I}\right) \cdot\left(\mathbf{v}_{s}-\mathbf{v}^{\bar{s}}\right)\right\rangle_{\Omega_{s}, \Omega}\right]\right\} \\
& -\sum_{\iota \in \mathcal{J}_{p}}\left\{\epsilon^{\iota} b^{\iota}-\frac{1}{\theta^{\bar{\iota}}}\left[\epsilon^{\iota} h^{\iota}+\left\langle\eta_{\iota} \frac{\mathrm{D}^{\bar{s}}\left(\theta_{\iota}-\theta^{\bar{\imath}}\right)}{\mathrm{D} t}\right\rangle_{\Omega_{\iota}, \Omega}\right]\right. \\
& \left.-\frac{1}{\theta^{\bar{\imath}}}\left\langle\rho_{\iota} \frac{\mathrm{D}^{\bar{s}}\left(\mu_{\iota}+\psi_{\iota}-\mu^{\bar{\imath}}-K_{E}^{\overline{\bar{\tau}}}-\psi^{\bar{\tau}}\right)}{\mathrm{D} t}\right\rangle_{\Omega_{\iota}, \Omega}\right\} \\
& -\epsilon^{w s} b^{w s}+\frac{1}{\theta^{\overline{\overline{w s}}}}\left[\epsilon^{w s} h^{w s}+\left\langle\eta_{w s} \frac{\mathrm{D}^{\prime s}\left(\theta_{w s}-\theta^{\overline{\overline{w s}}}\right)}{\mathrm{D} t}\right\rangle_{\Omega_{w s}, \Omega}\right] \\
& +\frac{1}{\theta^{\overline{\overline{w s}}}}\left\langle\rho_{w s} \frac{\mathrm{D}^{\prime s}\left(\mu_{w s}+\psi_{w s}-\mu^{\overline{w s}}-K_{E}^{\overline{\overline{w s}}}-\psi^{\overline{w s}}\right)}{\mathrm{D} t}\right\rangle_{\Omega_{w s}, \Omega} \\
& +\frac{\epsilon^{w}}{\theta^{\overline{\bar{w}}}}\left(\mathbf{t}^{\overline{\bar{w}}}+p^{w} \mathbf{I}\right): \mathbf{d}^{\overline{\bar{w}}}+\frac{\epsilon^{s}}{\theta^{\overline{\bar{s}}}}\left(\mathbf{t}^{\overline{\bar{s}}}-\mathbf{t}^{s}\right): \mathbf{d}^{\overline{\bar{s}}}
\end{aligned}
$$




$$
\begin{aligned}
& +\frac{\epsilon^{w s}}{\theta^{\overline{\overline{w s}}}}\left(\mathbf{t}^{\overline{\overline{w s}}}-\gamma^{w s} \mathbf{I}\right): \mathbf{d}^{\overline{\overline{w s}}}+\frac{\epsilon^{w} \mathbf{q}^{\overline{\bar{w}}}}{\left(\theta^{\overline{\bar{w}}}\right)^{2}} \cdot \nabla \theta^{\overline{\bar{w}}}+\frac{\epsilon^{w s} \mathbf{q}^{\overline{\overline{w s}}}}{\left(\theta^{\overline{\overline{w s}}}\right)^{2}} \cdot \nabla \theta^{\overline{\overline{w s}}} \\
& +\frac{1}{\left(\theta^{\bar{s}}\right)^{2}}\left[\epsilon^{s} \mathbf{q}^{\overline{\bar{s}}}-\left\langle\left(\mathbf{t}_{s}-\boldsymbol{\sigma}_{s}: \frac{\mathbf{C}_{s}}{j_{s}} \mathbf{I}\right) \cdot\left(\mathbf{v}_{s}-\mathbf{v}^{\bar{s}}\right)\right\rangle_{\Omega_{s}, \Omega}\right] \cdot \nabla \theta^{\overline{\bar{s}}} \\
& -\sum_{\iota \in \mathfrak{J}_{p}} \stackrel{w s \rightarrow}{M} \stackrel{1}{\theta^{\overline{\overline{w s}}}}\left[\left(\mu^{\bar{\imath}}+K_{E}^{\overline{\bar{c}}}+\psi^{\bar{\iota}}\right)-\left(\mu^{\overline{w s}}+K_{E}^{\overline{\overline{w s}}}+\psi^{\overline{w s}}\right)\right] \\
& -\frac{1}{\theta^{\bar{s}}}\left\langle\left[\mathbf{n}_{s} \cdot \mathbf{t}_{s} \cdot \mathbf{n}_{s}-\boldsymbol{\sigma}_{s}: \frac{\mathbf{C}_{s}}{j_{s}}\right] \mathbf{n}_{s} \cdot\left(\mathbf{v}_{s}-\mathbf{v}_{w s}\right)\right\rangle_{\Omega_{w s}, \Omega} \\
& +\sum_{\iota \in \mathcal{J}_{p}}\left\{{ }^{w s \rightarrow \iota}+\left(\frac{E^{\overline{\bar{\iota}}}}{\epsilon^{\iota} \rho^{\iota}}-\mu^{\bar{\tau}}\right) \stackrel{w s \rightarrow \iota}{M}\right. \\
& \left.+\mathbf{v}^{\bar{\tau}, \bar{s}} \cdot\left[\stackrel{w s \rightarrow \iota}{\mathbf{T}}+\left(\frac{\mathbf{v}^{\bar{\tau}, \bar{s}}-\mathbf{v}^{\overline{w s}, \bar{s}}}{2}\right) \stackrel{w s \rightarrow \iota}{M}\right]\right\}\left(\frac{1}{\theta^{\overline{\bar{\iota}}}}-\frac{1}{\theta^{\overline{\overline{w s}}}}\right) \\
& -\frac{1}{\theta^{\bar{w}}}\left\{\stackrel{w s \rightarrow w}{\mathbf{T}}+\left(\frac{\mathbf{v}^{\bar{w}, \bar{s}}-\mathbf{v}^{\overline{w s}, \bar{s}}}{2}\right) \stackrel{w s \rightarrow w}{M}+\epsilon^{w} \rho^{w} \mathbf{g}^{\bar{w}}\right. \\
& \left.+\epsilon^{w} \rho^{w} \nabla\left(\psi^{\bar{w}}+\mu^{\bar{w}}+K_{E}^{\overline{\bar{w}}}\right)-\nabla\left(\epsilon^{w} p^{w}\right)+\eta^{\overline{\bar{w}}} \nabla \theta^{\overline{\bar{w}}}\right\} \cdot \mathbf{v}^{\bar{w}, \bar{s}} \\
& +\frac{1}{\theta^{\overline{\overline{w s}}}}\left\{\sum_{\iota \in \mathcal{J}_{p}}\left[\stackrel{w s \rightarrow \iota}{\mathbf{T}}+\left(\frac{\mathbf{v}^{\bar{\imath}, \bar{s}}-\mathbf{v}^{\overline{w s}, \bar{s}}}{2}\right) \stackrel{w s \rightarrow \iota}{M}\right]-\epsilon^{w s} \rho^{w s} \mathbf{g}^{\overline{w s}}\right. \\
& \left.-\epsilon^{w s} \rho^{w s} \nabla\left(\mu^{\overline{w s}}+K_{E}^{\overline{\overline{w s}}}+\psi^{\overline{w s}}\right)-\eta^{\overline{\overline{w s}}} \nabla \theta^{\overline{\overline{w s}}}-\nabla\left(\epsilon^{w s} \gamma^{w s}\right)\right\} \cdot \mathbf{v}^{\overline{w s}, \bar{s}} \\
& -\frac{1}{\theta^{\bar{s}}}\left\langle\mathbf{n}_{s} \cdot \mathbf{t}_{s} \cdot \mathbf{l}^{\prime} \cdot\left(\mathbf{v}_{s}-\mathbf{v}^{\bar{s}}\right)\right\rangle_{\Omega_{w s}, \Omega}+\frac{1}{\theta^{\bar{s}}}\left\langle\left[\nabla \cdot \mathbf{t}_{s}-\nabla \boldsymbol{\sigma}_{s}: \frac{\mathbf{C}_{s}}{j_{s}}\right] \cdot\left(\mathbf{v}_{s}-\mathbf{v}^{\bar{s}}\right)\right\rangle_{\Omega_{s}, \Omega} \\
& -\frac{1}{\theta^{\overline{\overline{w s}}}}\left\langle\left[p_{w}+\mathbf{n}_{s} \cdot \mathbf{t}_{s} \cdot \mathbf{n}_{s}+\gamma_{w s} \nabla^{\prime} \cdot \mathbf{n}_{s}-\rho_{w s} \mathbf{g}_{w s} \cdot \mathbf{n}_{s}\right]\left(\mathbf{v}_{w s}-\mathbf{v}^{\bar{s}}\right) \cdot \mathbf{n}_{s}\right\rangle_{\Omega_{w s}, \Omega} \\
& -\left\langle p_{w}\left(\mathbf{v}_{w s}-\mathbf{v}^{\bar{s}}\right) \cdot \mathbf{n}_{s}\right\rangle_{\Omega_{w s}, \Omega}\left(\frac{1}{\theta^{\overline{\bar{w}}}}-\frac{1}{\theta^{\overline{\overline{w s}}}}\right) \\
& -\left\langle\mathbf{n}_{s} \cdot \mathbf{t}_{s} \cdot \mathbf{n}_{s}\left(\mathbf{v}_{w s}-\mathbf{v}^{\bar{s}}\right) \cdot \mathbf{n}_{s}\right\rangle_{\Omega_{w s}, \Omega}\left(\frac{1}{\theta^{\bar{s}}}-\frac{1}{\theta^{\overline{w s}}}\right) \\
& +\frac{1}{\theta^{\overline{\overline{w s}}}}\left[\nabla \cdot\left\langle\mathbf{n}_{s} \mathbf{n}_{s} \cdot\left(\mathbf{v}_{w s}-\mathbf{v}^{\bar{s}}\right) \gamma_{w s}\right\rangle_{\Omega_{w s}, \Omega}+\left\langle\mathbf{n}_{s} \mathbf{n}_{s} \gamma_{w s}\right\rangle_{\Omega_{w s}, \Omega}: \mathbf{d}^{\overline{\bar{s}}}\right] \\
& +\frac{1}{\theta^{\overline{\overline{w s}}}} \nabla \theta^{\overline{\overline{w s}}} \cdot\left\langle\mathbf{n}_{w} \mathbf{n}_{w} \cdot\left(\mathbf{v}_{w s}-\mathbf{v}^{\bar{s}}\right) \eta_{w s}\right\rangle_{\Omega_{w s}, \Omega} \\
& +\frac{1}{\theta^{\overline{\overline{w s}}}} \nabla\left(\mu^{\overline{w s}}+K_{E}^{\overline{\overline{w s}}}+\psi^{\overline{w s}}\right) \cdot\left\langle\mathbf{n}_{w} \mathbf{n}_{w} \cdot\left(\mathbf{v}_{w s}-\mathbf{v}^{\bar{s}}\right) \rho_{w s}\right\rangle_{\Omega_{w s}, \Omega}=\Lambda \geq 0
\end{aligned}
$$


Eqn (68) is the form of the CEI that we obtain by rearranging terms in an effort to obtain groupings that correspond to the form of eqn (56). Although many of the terms are in the needed form of a product for which each factor is zero at equilibrium, some of the terms must be rearranged further. The form of eqn (68) is impacted by the choice of the microscale thermodynamic functional dependence, a choice which is reasonable but not unique. No mathematical approximations have been employed in obtaining this equation from eqn (57). This is an important equation, because it provides a starting point for the formulation of a range of complete closed models for single-phase flow. The steps taken from here to obtain those models require approximations appropriate for the physical system under consideration and may take different forms depending on the approximations employed.

\section{Simplified Entropy Inequality}

Our goal is to produce a simplified EI (SEI) from the CEI that can be used to guide the formulation of closed models. We emphasize that the steps needed to derive the SEI from the CEI are approximate in nature. If better approximations become available, or should an exact relation be derived for a particular system, alternatives to the approximations used here may be employed to produce the SEI.

Although many of the terms in eqn (68) are grouped into force-flux pairs, some terms are not yet in this form. The first three groups in the equation require special attention and will be dealt with in due course. Of particular note in obtaining an equation with force-flux pairs are the terms in the averaging operators that involve integrals of $\mathbf{n}_{w} \mathbf{n}_{w}$. Since these terms are related to 
the orientation of the $w s$ interface, we refer to them collectively as geometric terms.

We define the geometric tensor for the ws interface, $\mathbf{G}^{w s}$, as

$$
\epsilon^{w s} \mathbf{G}^{w s}=\left\langle\mathbf{G}_{w s}\right\rangle_{\Omega_{w s}, \Omega}=\left\langle\mathbf{n}_{w} \mathbf{n}_{w}\right\rangle_{\Omega_{w s}, \Omega}
$$

For a single-phase system, $\mathbf{G}^{w s}$ is a descriptor of the orientation of the solid grains. When the orientation of the surface of the solid grains is random, $\mathbf{G}^{w s}=$ $\mathbf{I}$ 3. When the principal directions of solid grain orientation are aligned with the coordinate system, $\mathbf{G}^{w s}$ is a diagonal tensor. The trace of the geometric tensor, denoted $\operatorname{Tr}\left(\mathbf{G}^{w s}\right)$, is equal to 1 and is an invariant of $\mathbf{G}^{w s}$. Knowledge of the microscale is sufficient to compute $\mathbf{G}^{\text {ws }}$ without error and thereby test the macroscale models derived from this theory.

In general, $\mathbf{G}_{w s}$ appears within averaging operators as a product involving other terms. As a first approximation, we will assume that these product terms are independent, such that the orientation of the interface does not depend upon interfacial tension or velocities. Applying these approximations and similar product-splitting integral approximations, and relating changes in $\epsilon^{s}$ to changes in $\epsilon^{w s}$, we reduce the CEI to an SEI of the form

$$
\begin{gathered}
-\nabla \cdot\left[\epsilon^{w}\left(\boldsymbol{\varphi}^{\overline{\bar{w}}}-\frac{\mathbf{q}^{\overline{\bar{w}}}}{\theta^{\overline{\bar{w}}}}\right)\right]-\nabla \cdot\left[\epsilon^{w s}\left(\boldsymbol{\varphi}^{\overline{\overline{w s}}}-\frac{\mathbf{q}^{\overline{\bar{w}}}}{\theta^{\overline{\overline{w s}}}}\right)\right] \\
-\nabla \cdot\left\{\epsilon^{s} \boldsymbol{\varphi}^{\overline{\bar{s}}}-\frac{1}{\theta^{\bar{s}}}\left[\epsilon^{s} \mathbf{q}^{\overline{\bar{s}}}-\left\langle\left(\mathbf{t}_{s}-\boldsymbol{\sigma}_{s}: \frac{\mathbf{C}_{s}}{j_{s}} \mathbf{l}\right) \cdot\left(\mathbf{v}_{s}-\mathbf{v}^{\bar{s}}\right)\right\rangle_{\Omega_{s}, \Omega}\right]\right\} \\
-\sum_{\iota \in J_{p}}\left\{\epsilon^{\iota} b^{\iota}-\frac{1}{\theta^{\bar{\iota}}}\left[\epsilon^{\iota} h^{\iota}+\left\langle\eta_{\iota} \frac{\mathrm{D}^{\bar{s}}\left(\theta_{\iota}-\theta^{\bar{\imath}}\right)}{\mathrm{D} t}\right\rangle_{\Omega_{\iota}, \Omega}\right]\right. \\
\left.-\frac{1}{\theta^{\bar{\iota}}}\left\langle\rho_{\iota} \frac{\mathrm{D}^{\bar{s}}\left(\mu_{\iota}+\psi_{\iota}-\mu^{\bar{\iota}}-K_{E}^{\overline{\bar{\imath}}}-\psi^{\bar{\iota}}\right)}{\mathrm{D} t}\right\rangle_{\Omega_{\iota}, \Omega}\right\}
\end{gathered}
$$




$$
\begin{aligned}
& -\epsilon^{w s} b^{w s}+\frac{1}{\theta^{\overline{\overline{w s}}}}\left[\epsilon^{w s} h^{w s}+\left\langle\eta_{w s} \frac{\mathrm{D}^{\prime s}\left(\theta_{w s}-\theta^{\overline{\overline{w s}}}\right)}{\mathrm{D} t}\right\rangle_{\Omega_{w s}, \Omega}\right] \\
& +\frac{1}{\theta^{\overline{w s}}}\left\langle\rho_{w s} \frac{\mathrm{D}^{\prime s}\left(\mu_{w s}+\psi_{w s}-\mu^{\overline{w s}}-K_{E}^{\overline{\overline{w s}}}-\psi^{\overline{w s}}\right)}{\mathrm{D} t}\right\rangle_{\Omega_{w s}, \Omega} \\
& +\frac{\epsilon^{w}}{\theta^{\overline{\bar{w}}}}\left(\mathbf{t}^{\overline{\bar{w}}}+p^{w} \mathbf{I}\right): \mathbf{d}^{\overline{\bar{w}}}+\frac{\epsilon^{s}}{\theta^{\overline{\bar{s}}}}\left(\mathbf{t}^{\overline{\bar{s}}}-\mathbf{t}^{s}\right): \mathbf{d}^{\overline{\bar{s}}} \\
& +\frac{\epsilon^{w s}}{\theta^{\overline{\overline{w s}}}}\left[\mathbf{t}^{\overline{\overline{w s}}}-\gamma^{w s}\left(\mathbf{I}-\mathbf{G}^{w s}\right)\right]: \mathbf{d}^{\overline{\overline{w s}}}+\frac{\epsilon^{w} \mathbf{q}^{\overline{\bar{w}}}}{\left(\theta^{\overline{\bar{w}}}\right)^{2}} \cdot \nabla \theta^{\overline{\bar{w}}}+\frac{\epsilon^{w s} \mathbf{q}^{\overline{\overline{w s}}}}{\left(\theta^{\overline{\overline{w s}}}\right)^{2}} \cdot \nabla \theta^{\overline{\overline{w s}}} \\
& +\frac{1}{\left(\theta^{\bar{s}}\right)^{2}}\left[\epsilon^{s} \mathbf{q}^{\overline{\bar{s}}}-\left\langle\left(\mathbf{t}_{s}-\boldsymbol{\sigma}_{s}: \frac{\mathbf{C}_{s}}{j_{s}} \mathbf{I}\right) \cdot\left(\mathbf{v}_{s}-\mathbf{v}^{\bar{s}}\right)\right\rangle_{\Omega_{s}, \Omega}\right] \cdot \nabla \theta^{\overline{\bar{s}}} \\
& -\stackrel{w s \rightarrow w}{M} \frac{1}{\theta^{\overline{\overline{w s}}}}\left[\left(\mu^{\bar{w}}+K_{E}^{\overline{\bar{w}}}+\psi^{\bar{w}}\right)-\left(\mu^{\overline{w s}}+K_{E}^{\overline{\overline{w s}}}+\psi^{\overline{w s}}\right)\right] \\
& -{ }^{w s} \rightarrow s \frac{1}{\theta^{\overline{w s}}}\left\{\left(\mu^{\bar{s}}+K_{E}^{\overline{\bar{s}}}+\psi^{\bar{s}}\right)+\left\langle\frac{\boldsymbol{\sigma}_{s}}{\rho_{s}}: \frac{\mathbf{C}_{s}}{j_{s}}-\frac{1}{\rho_{s}} \mathbf{n}_{s} \cdot \mathbf{t}_{s} \cdot \mathbf{n}_{s}\right\rangle_{\Omega_{w s}, \Omega_{w s}}\right. \\
& \left.-\left(\mu^{\overline{w s}}+K_{E}^{\overline{\overline{w s}}}+\psi^{\overline{w s}}\right)\right\} \\
& -\frac{1}{\theta^{\overline{\bar{w}}}}\left\{\stackrel{w s \rightarrow w}{\mathbf{T}}+\left(\frac{\mathbf{v}^{\bar{w}, \bar{s}}-\mathbf{v}^{\overline{w s}, \bar{s}}}{2}\right) \stackrel{w s \rightarrow w}{M}+\epsilon^{w} \rho^{w} \mathbf{g}^{\bar{w}}\right. \\
& \left.+\epsilon^{w} \rho^{w} \nabla\left(\psi^{\bar{w}}+\mu^{\bar{w}}+K_{E}^{\overline{\bar{w}}}\right)-\nabla\left(\epsilon^{w} p^{w}\right)+\eta^{\overline{\bar{w}}} \nabla \theta^{\overline{\bar{w}}}\right\} \cdot \mathbf{v}^{\bar{w}, \bar{s}} \\
& +\frac{1}{\theta^{\overline{\overline{w s}}}}\left\{\sum_{\iota \in \mathcal{J}_{p}}\left[\stackrel{w s \rightarrow \iota}{\mathbf{T}}+\left(\frac{\mathbf{v}^{\bar{\imath}, \bar{s}}-\mathbf{v}^{\overline{w s}, \bar{s}}}{2}\right) \stackrel{w s \rightarrow \iota}{M}\right]-\epsilon^{w s} \rho^{w s} \mathbf{g}^{\overline{w s}}\right. \\
& -\epsilon^{w s} \rho^{w s}\left(\mathbf{I}-\mathbf{G}^{w s}\right) \cdot \nabla\left(\mu^{\overline{w s}}+K_{E}^{\overline{\overline{w s}}}+\psi^{\overline{w s}}\right) \\
& \left.-\eta^{\overline{\overline{w s}}}\left(\mathbf{I}-\mathbf{G}^{w s}\right) \cdot \nabla \theta^{\overline{\overline{w s}}}-\nabla \cdot\left[\epsilon^{w s} \gamma^{w s}\left(\mathbf{I}-\mathbf{G}^{w s}\right)\right]\right\} \cdot \mathbf{v}^{\overline{w s}, \bar{s}} \\
& -\frac{1}{\theta^{\overline{w s}}}\left\langle p_{w}+\mathbf{n}_{s} \cdot \mathbf{t}_{s} \cdot \mathbf{n}_{s}+\gamma_{w s} \nabla^{\prime} \cdot \mathbf{n}_{s}-\rho_{w s} \mathbf{g}_{w s} \cdot \mathbf{n}_{s}\right\rangle_{\Omega_{w s}, \Omega_{w s}} \frac{\mathrm{D}^{\bar{s}} \epsilon^{s}}{\mathrm{D} t}
\end{aligned}
$$

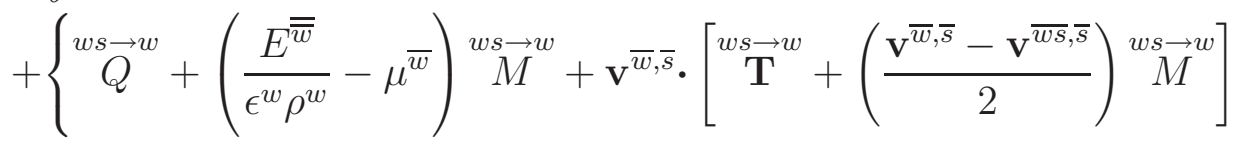

$$
\begin{aligned}
& \left.-\left\langle p_{w}\right\rangle_{\Omega_{w s}, \Omega_{w s}} \frac{\mathrm{D}^{\bar{s}} \epsilon^{s}}{\mathrm{D} t}\right\}\left(\frac{1}{\theta^{\overline{\bar{w}}}}-\frac{1}{\theta^{\overline{\overline{w s}}}}\right)
\end{aligned}
$$

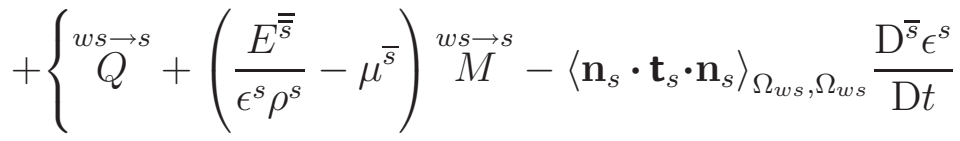




$$
\begin{array}{r}
\left.-\left\langle\frac{\boldsymbol{\sigma}_{s}}{\rho_{s}}: \frac{\mathbf{C}_{s}}{j_{s}}-\frac{1}{\rho_{s}} \mathbf{n}_{s} \cdot \mathbf{t}_{s} \cdot \mathbf{n}_{s}\right\rangle_{\Omega_{w s}, \Omega_{w s}} \stackrel{w}{M}^{s}\right\}\left(\frac{1}{\theta^{\overline{\bar{s}}}}-\frac{1}{\theta^{\overline{\overline{w s}}}}\right) \\
-\frac{1}{\theta^{\bar{s}}}\left\langle\mathbf{n}_{s} \cdot \mathbf{t}_{s} \cdot \mathbf{I}^{\prime} \cdot\left(\mathbf{v}_{s}-\mathbf{v}^{\bar{s}}\right)\right\rangle_{\Omega_{w s}, \Omega} \\
+\frac{1}{\theta^{\overline{\bar{s}}}}\left\langle\left(\nabla \cdot \mathbf{t}_{s}-\nabla \boldsymbol{\sigma}_{s}: \frac{\mathbf{C}_{s}}{j_{s}}\right) \cdot\left(\mathbf{v}_{s}-\mathbf{v}^{\bar{s}}\right)\right\rangle_{\Omega_{s}, \Omega}=\Lambda \geq 0
\end{array}
$$

When we developed the EI, we noted the importance of obtaining a relation between the entropy and other variables that appear in conservation equations. Additionally, the entropy equation has a non-advective flux and a source term that also need to be related to other variables that appear in the conservation equations. In particular, the non-advective energy and heat fluxes need to be related as do the entropy and energy source terms. To make this connection, the systems of concern in this work are considered to be macroscopically simple, which we define as systems in which the relation between the entropy and heat fluxes can be written without introduction of any new constitutive variables and, similarly, the relation between the entropy and energy source terms can be written without additional constitutive forms. The conditions employed are based on eqn (70) but are also consistent with the identification of simple systems at the microscale [e.g., 8, 13]. For macroscopically simple systems, the entropy and heat fluxes are related by

$$
\epsilon^{\iota} \boldsymbol{\varphi}^{\overline{\bar{\iota}}}-\frac{1}{\theta^{\overline{\bar{\iota}}}} \epsilon^{\iota} \mathbf{q}^{\overline{\bar{\iota}}}=0, \quad \text { for } \iota \in\{w, w s\}
$$

and

$$
\epsilon^{s} \boldsymbol{\varphi}^{\overline{\bar{s}}}-\frac{1}{\theta^{\overline{\bar{s}}}}\left[\epsilon^{s} \mathbf{q}^{\overline{\bar{s}}}-\left\langle\left(\mathbf{t}_{s}-\boldsymbol{\sigma}_{s}: \frac{\mathbf{C}_{s}}{j_{s}} \mathbf{l}\right) \cdot\left(\mathbf{v}_{s}-\mathbf{v}^{\bar{s}}\right)\right\rangle_{\Omega_{s}, \Omega}\right]=0
$$

If a more complex relationship is found necessary, the right side of these expressions can be set to some non-zero constitutive function of the force variables in the SEI. 
Similarly, we relate the energy and entropy source terms in consideration of eqn (70) according to

$$
\begin{array}{r}
\epsilon^{\iota} b^{\iota}-\frac{1}{\theta^{\overline{\bar{l}}}}\left[\epsilon^{\iota} h^{\iota}+\left\langle\eta_{\iota} \frac{\mathrm{D}^{\bar{s}}\left(\theta_{\iota}-\theta^{\overline{\bar{\tau}}}\right)}{\mathrm{D} t}+\rho_{\iota} \frac{\mathrm{D}^{\bar{s}}\left(\mu_{\iota}+\psi_{\iota}-\mu^{\bar{\tau}}-K_{E}^{\overline{\bar{\tau}}}-\psi^{\bar{\tau}}\right)}{\mathrm{D} t}\right\rangle_{\Omega_{\iota}, \Omega}\right]=0 \\
\text { for } \iota \in \mathcal{J}_{p} \quad(72 \mathrm{a})
\end{array}
$$

and

$$
\begin{aligned}
& \epsilon^{w s} b^{w s}-\frac{1}{\theta^{\overline{\overline{w s}}}}\left[\epsilon^{w s} h^{w s}+\left\langle\eta_{w s} \frac{\mathrm{D}^{\prime s}\left(\theta_{w s}-\theta^{\overline{\overline{w s}}}\right)}{\mathrm{D} t}\right\rangle_{\Omega_{w s}, \Omega}\right. \\
& \left.+\left\langle\rho_{w s} \frac{\mathrm{D}^{\prime s}\left(\mu_{w s}+\psi_{w s}-\mu^{\overline{w s}}-K_{E}^{\overline{\overline{w s}}}-\psi^{\overline{w s}}\right)}{\mathrm{D} t}\right\rangle_{\Omega_{w s}, \Omega}\right]=0
\end{aligned}
$$

We now make a final set of approximations to simplify eqn (70). The last two terms on the left side involve averages of products of microscale quantities for which the factors are all zero at equilibrium. These terms all involve the solid phase. We will assume that the solid-phase deformation is slow enough that these expressions involve products of small terms that may be considered to satisfy quasi-equilibrium conditions and be neglected even away from equilibrium. With these conditions applied, the SEI takes the still rather general form

$$
\begin{aligned}
& \frac{\epsilon^{w}}{\theta^{\overline{\bar{w}}}}\left(\mathbf{t}^{\overline{\bar{w}}}+p^{w} \mathbf{I}\right): \mathbf{d}^{\overline{\bar{w}}}+\frac{\epsilon^{s}}{\theta^{\overline{\bar{s}}}}\left(\mathbf{t}^{\overline{\bar{s}}}-\mathbf{t}^{s}\right): \mathbf{d}^{\overline{\bar{s}}} \\
& +\frac{\epsilon^{w s}}{\theta^{\overline{\overline{w s}}}}\left[\mathbf{t}^{\overline{\overline{w s}}}-\gamma^{w s}\left(\mathbf{I}-\mathbf{G}^{w s}\right)\right]: \mathbf{d}^{\overline{\overline{w s}}}+\frac{\epsilon^{w} \mathbf{q}^{\overline{\bar{w}}}}{\left(\theta^{\overline{\bar{w}}}\right)^{2}} \cdot \nabla \theta^{\overline{\bar{w}}}+\frac{\epsilon^{w s} \mathbf{q}^{\overline{\overline{w s}}}}{\left(\theta^{\overline{\overline{w s}}}\right)^{2}} \cdot \nabla \theta^{\overline{\overline{w s}}} \\
& +\frac{1}{\left(\theta^{\bar{s}}\right)^{2}}\left[\epsilon^{s} \mathbf{q}^{\overline{\bar{s}}}-\left\langle\left(\mathbf{t}_{s}-\boldsymbol{\sigma}_{s}: \frac{\mathbf{C}_{s}}{j_{s}} \mathbf{I}\right) \cdot\left(\mathbf{v}_{s}-\mathbf{v}^{\bar{s}}\right)\right\rangle_{\Omega_{s}, \Omega}\right] \cdot \nabla \theta^{\overline{\bar{s}}} \\
& -{ }^{w s} \stackrel{w}{w}^{w} \frac{1}{\theta^{\overline{w s}}}\left[\left(\mu^{\bar{w}}+K_{E}^{\overline{\bar{w}}}+\psi^{\bar{w}}\right)-\left(\mu^{\overline{w s}}+K_{E}^{\overline{\overline{w s}}}+\psi^{\overline{w s}}\right)\right]
\end{aligned}
$$




$$
\begin{aligned}
& -{ }^{w s} \rightarrow s \frac{1}{\theta^{\overline{w s}}}\left\{\left(\mu^{\bar{s}}+K_{E}^{\overline{\bar{s}}}+\psi^{\bar{s}}\right)+\left\langle\frac{\boldsymbol{\sigma}_{s}}{\rho_{s}}: \frac{\mathbf{C}_{s}}{j_{s}}-\frac{1}{\rho_{s}} \mathbf{n}_{s} \cdot \mathbf{t}_{s} \cdot \mathbf{n}_{s}\right\rangle_{\Omega_{w s}, \Omega_{w s}}\right. \\
& \left.-\left(\mu^{\overline{w s}}+K_{E}^{\overline{\overline{w s}}}+\psi^{\overline{w s}}\right)\right\} \\
& -\frac{1}{\theta^{\bar{w}}}\left\{\stackrel{w s \rightarrow w}{\mathbf{T}}+\left(\frac{\mathbf{v}^{\bar{w}, \bar{s}}-\mathbf{v}^{\overline{w s}, \bar{s}}}{2}\right) \stackrel{w s \rightarrow w}{M}+\epsilon^{w} \rho^{w} \mathbf{g}^{\bar{w}}\right. \\
& \left.+\epsilon^{w} \rho^{w} \nabla\left(\psi^{\bar{w}}+\mu^{\bar{w}}+K_{E}^{\overline{\bar{w}}}\right)-\nabla\left(\epsilon^{w} p^{w}\right)+\eta^{\overline{\bar{w}}} \nabla \theta^{\overline{\bar{w}}}\right\} \cdot \mathbf{v}^{\bar{w}, \bar{s}} \\
& +\frac{1}{\theta^{\overline{\overline{w s}}}}\left\{\sum_{\iota \in \mathcal{J}_{p}}\left[\stackrel{w s \rightarrow \iota}{\mathbf{T}}+\left(\frac{\mathbf{v}^{\bar{\imath}, \bar{s}}-\mathbf{v}^{\overline{w s}, \bar{s}}}{2}\right) \stackrel{w s \rightarrow \iota}{M}\right]-\epsilon^{w s} \rho^{w s} \mathbf{g}^{\overline{w s}}\right. \\
& -\epsilon^{w s} \rho^{w s}\left(\mathbf{I}-\mathbf{G}^{w s}\right) \cdot \nabla\left(\mu^{\overline{w s}}+K_{E}^{\overline{\overline{w s}}}+\psi^{\overline{w s}}\right) \\
& \left.-\eta^{\overline{\overline{w s}}}\left(\mathbf{I}-\mathbf{G}^{w s}\right) \cdot \nabla \theta^{\overline{\overline{w s}}}-\nabla \cdot\left[\epsilon^{w s} \gamma^{w s}\left(\mathbf{I}-\mathbf{G}^{w s}\right)\right]\right\} \cdot \mathbf{v}^{\overline{w s}, \bar{s}} \\
& -\frac{1}{\theta^{\overline{\overline{w s}}}}\left\langle p_{w}+\mathbf{n}_{s} \cdot \mathbf{t}_{s} \cdot \mathbf{n}_{s}+\gamma_{w s} \nabla^{\prime} \cdot \mathbf{n}_{s}-\rho_{w s} \mathbf{g}_{w s} \cdot \mathbf{n}_{s}\right\rangle_{\Omega_{w s}, \Omega_{w s}} \frac{\mathrm{D}^{\bar{s}} \epsilon^{s}}{\mathrm{D} t}
\end{aligned}
$$

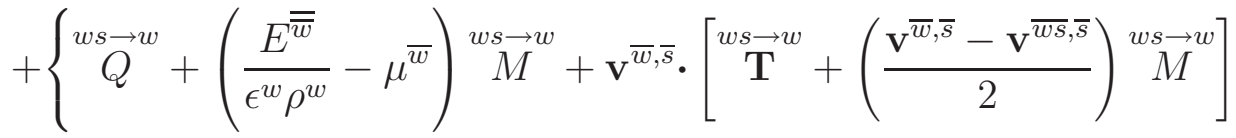

$$
\begin{aligned}
& \left.-\left\langle p_{w}\right\rangle_{\Omega_{w s}, \Omega_{w s}} \frac{\mathrm{D}^{\bar{s}} \epsilon^{s}}{\mathrm{D} t}\right\}\left(\frac{1}{\theta^{\overline{\bar{w}}}}-\frac{1}{\theta^{\overline{\overline{w s}}}}\right)
\end{aligned}
$$

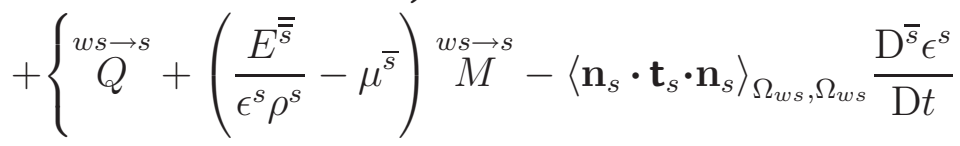

$$
\begin{aligned}
& \left.-\left\langle\frac{\boldsymbol{\sigma}_{s}}{\rho_{s}}: \frac{\mathbf{C}_{s}}{j_{s}}-\frac{1}{\rho_{s}} \mathbf{n}_{s} \cdot \mathbf{t}_{s} \cdot \mathbf{n}_{s}\right\rangle_{\Omega_{w s}, \Omega_{w s}} \stackrel{w s \rightarrow s}{M}\right\}\left(\frac{1}{\theta^{\bar{s}}}-\frac{1}{\theta^{\overline{\overline{w s}}}}\right)=\Lambda \geq 0
\end{aligned}
$$

This equation has been arranged such that it is a sum of products of fluxes and forces with the forces being the second term in each product. Knowledge that the forces are zero at equilibrium comes from the definition of thermodynamic equilibrium conditions summarized in eqns (44)-(51). The fluxes corresponding to the forces are taken to be zero at equilibrium in this work; a detailed analysis to confirm these conclusions could be undertaken, but we have not done so. 


\section{Closure Relation Approximations}

The surviving terms in eqn (73) consist of products of thermodynamic fluxes and forces that satisfy the conditions summarized in Postulate 1. Because all forces and fluxes vanish at equilibrium, the non-negative property of $\Lambda$ may be exploited to obtain closed form approximations for the fluxes. The closure relations are obtained by expressing the flux terms as functions of the forces. Such approximations must yield non-negative quantities for the product of a flux and its conjugate force, but the forms are not unique. They must, however, be appropriate for the system under consideration and describe the physics of interest and importance. For example, the zero-order approximation is that a flux is identically zero at all locations in space and at all times. The simplest linear approximation expresses a flux as a linear function of its conjugate force. So-called cross-coupled flux approximations express the flux as a linear function of all of the forces in the system. Higher order approximations are necessary in some cases. Because the objective of this work is to demonstrate the TCAT approach and lay an operational foundation upon which future work can be built, we will assume a relatively simple form for the closure relations - zero or first-order approximations for each flux as a function of only its conjugate force with the approximation order relating to the phenomena we wish to describe.

The flux-force combinations consisting of products of fluxes involving stress

tensors with rate of strain tensors $\mathbf{d}^{\overline{\bar{\iota}}}$ as the conjugate forces are approximated using a zero-order approximation. The fluxes are considered to be independent of $\mathbf{d}^{\overline{\bar{t}}}$ and therefore, to ensure that eqn (73) is satisfied for all independent variations, these forces must be zero and the constitutive forms obtained are 


$$
\begin{aligned}
& \mathbf{t}^{\overline{\bar{w}}}+p^{w} \mathbf{I}=0 \\
& \mathbf{t}^{\overline{\bar{s}}}-\mathbf{t}^{s}=0 \\
& \mathbf{t}^{\overline{\overline{w s}}}-\gamma^{w s}\left(\mathbf{I}-\mathbf{G}^{w s}\right)=0
\end{aligned}
$$

These approximate closure relations amount to an assumption of macroscopically inviscid flow or flow where the rate of strain is negligible. In either case, the flux-force combinations involving the rate of strain tensor do not produce any entropy. This is assured because the terms multiplying the rate of strain tensors are zero at all spatial locations and times. Relaxing this assumption, such that the force vanishes only at equilibrium but may be non-zero away from equilibrium, leads to an alternative constitutive form of the stress tensor that will involve entropy production when the rate of strain is non-zero (i.e., away from equilibrium). In the simplest extension, the stress tensors could be expressed as linear functions of the corresponding rates of strain. For some systems, such an alternative formulation might be appropriate; but in most instances, the stress tensor forms of eqns (74)-(76) describe the system well.

Next consider the terms involving $\mathbf{q}^{\overline{\bar{\imath}}}$, the heat flux vectors. At equilibrium both this flux and its conjugate force must vanish for the inequality to hold. A linear approximation for the closure relation of the flux is

$$
\mathbf{q}^{\overline{\bar{\iota}}}=\hat{\mathbf{K}}_{q}^{\iota} \cdot \nabla \theta^{\overline{\bar{\iota}}}, \quad \text { for } \iota \in\{w, w s\}
$$

and for the solid phase

$$
\epsilon^{s} \mathbf{q}^{\overline{\bar{s}}}-\left\langle\left(\mathbf{t}_{s}-\boldsymbol{\sigma}_{s}: \frac{\mathbf{C}_{s}}{j_{s}} \mathbf{I}\right) \cdot\left(\mathbf{v}_{s}-\mathbf{v}^{\bar{s}}\right)\right\rangle_{\Omega_{s}, \Omega}=\epsilon^{s} \hat{\mathbf{K}}_{q}^{s} \cdot \nabla \theta^{\overline{\bar{s}}}
$$

where the coefficients $\hat{\mathbf{K}}_{q}^{\iota}$ in these equations are second-rank, symmetric, positive semi-definite heat conduction tensors. The hat notation is used to denote all model parameters. If an entity is isotropic, its heat conduction tensor is a 
scalar multiplying a unit tensor.

Closure approximations can be written for the mass exchange terms by approximating each mass exchange flux as linearly dependent upon its conjugate force in the flux-force pair. This approximation yields

$$
\stackrel{w s \rightarrow w}{M}=\hat{k}_{m}^{w}\left[\left(\mu^{\overline{w s}}+K_{E}^{\overline{\overline{w s}}}+\psi^{\overline{w s}}\right)-\left(\mu^{\bar{w}}+K_{E}^{\overline{\bar{w}}}+\psi^{\bar{w}}\right)\right]
$$

and

$$
\begin{aligned}
& \stackrel{w s \rightarrow s}{M}=\hat{k}_{m}^{s}\left[\left(\mu^{\overline{w s}}+K_{E}^{\overline{\overline{w s}}}+\psi^{\overline{w s}}\right)\right. \\
& \left.\quad-\left(\mu^{\bar{s}}+K_{E}^{\overline{\bar{s}}}+\psi^{\bar{s}}+\frac{\boldsymbol{\sigma}^{s}}{\rho^{s}}: \frac{\mathbf{C}^{s}}{j^{s}}-\left\langle\frac{1}{\rho_{s}} \mathbf{n}_{s} \cdot \mathbf{t}_{s} \cdot \mathbf{n}_{s}\right\rangle_{\Omega_{w s}, \Omega_{w s}}\right)\right]
\end{aligned}
$$

where $\hat{k}_{m}^{w}$ and $\hat{k}_{m}^{s}$ are non-negative interfacial mass transfer parameters.

Next, consider the flux-force pair involving momentum transfer of the $w$ phase to the ws interface in the flux and $\mathbf{v}^{\bar{w}, \bar{s}}$ as the force. The linearized force expression is

$$
\begin{aligned}
& \stackrel{w s \rightarrow w}{\mathbf{T}}+\left(\frac{\mathbf{v}^{\bar{w}, \bar{s}}-\mathbf{v}^{\overline{w s}, \bar{s}}}{2}\right) \stackrel{w s \rightarrow w}{M}+\epsilon^{w} \rho^{w} \mathbf{g}^{\bar{w}}+\epsilon^{w} \rho^{w} \nabla\left(\psi^{\bar{w}}+\mu^{\bar{w}}+K_{E}^{\overline{\bar{w}}}\right) \\
& \quad-\nabla\left(\epsilon^{w} p^{w}\right)+\eta^{\overline{\bar{w}}} \nabla \theta^{\overline{\bar{w}}}=-\hat{\mathbf{R}}^{w} \cdot \mathbf{v}^{\bar{w}, \bar{s}}
\end{aligned}
$$

For momentum transfer to the ws entity a linear approximation can be formulated from the flux that multiplies the force $\mathbf{v}^{\overline{w s}, \bar{s}}$

$$
\begin{aligned}
& \sum_{\iota \in \mathcal{J}_{p}}\left[\stackrel{w s \rightarrow \iota}{\mathbf{T}}+\left(\frac{\mathbf{v}^{\bar{\imath}, \bar{s}}-\mathbf{v}^{\overline{w s}, \bar{s}}}{2}\right) \stackrel{w s \rightarrow^{\iota}}{M^{\imath}}\right]-\epsilon^{w s} \rho^{w s} \mathbf{g}^{\overline{w s}} \\
& -\epsilon^{w s} \rho^{w s}\left(\mathbf{I}-\mathbf{G}^{w s}\right) \cdot \nabla\left(\mu^{\overline{w s}}+K_{E}^{\overline{\overline{w s}}}+\psi^{\overline{w s}}\right) \\
& -\eta^{\overline{\overline{w s}}}\left(\mathbf{I}-\mathbf{G}^{w s}\right) \cdot \nabla \theta^{\overline{\overline{w s}}}-\nabla \cdot\left[\epsilon^{w s} \gamma^{w s}\left(\mathbf{I}-\mathbf{G}^{w s}\right)\right]=\hat{\mathbf{R}}^{w s} \cdot \mathbf{v}^{\overline{w s}, \bar{s}}
\end{aligned}
$$

In these equations, $\hat{\mathbf{R}}^{w}$ and $\hat{\mathbf{R}}^{w s}$ are symmetric, positive semi-definite secondrank momentum resistance tensors that simplify to scaled unit tensors under 
conditions of entity isotropy.

The material derivative of the solid-phase volume fraction is considered a force term for a corresponding flux term that was shown to vanish at equilibrium. Thus both terms vanish at equilibrium, and the linearized force is expressed as

$$
-\left\langle p_{w}+\mathbf{n}_{s} \cdot \mathbf{t}_{s} \cdot \mathbf{n}_{s}+\gamma_{w s} \nabla^{\prime} \cdot \mathbf{n}_{s}-\rho_{w s} \mathbf{g}_{w s} \cdot \mathbf{n}_{s}\right\rangle_{\Omega_{w s}, \Omega_{w s}}=\hat{c} \frac{\mathrm{D}^{\bar{s}} \epsilon^{s}}{\mathrm{D} t}
$$

where $\hat{c}$ is a non-negative compressibility parameter.

Next we consider the energy exchange terms from the interface to each phase involving forces multiplied by inverse temperature differences. The two energy exchange expressions my be linearized in terms of their conjugate forces to obtain

$$
\begin{aligned}
\stackrel{w s \rightarrow w}{Q}+ & \left(\frac{E^{\overline{\bar{w}}}}{\epsilon^{w} \rho^{w}}-\mu^{\bar{w}}\right) \stackrel{w s \rightarrow w}{M}+\mathbf{v}^{\bar{w}, \bar{s}} \cdot\left[\begin{array}{c}
w s \rightarrow w \\
\mathbf{T}
\end{array}+\left(\frac{\mathbf{v}^{\bar{w}, \bar{s}}-\mathbf{v}^{\overline{w s}, \bar{s}}}{2}\right) \stackrel{w s \rightarrow w}{M}\right] \\
& -\left\langle p_{w}\right\rangle_{\Omega_{w s}, \Omega_{w s}} \frac{\mathrm{D}^{\bar{s}} \epsilon^{s}}{\mathrm{D} t}=\hat{k}_{q}^{w}\left(\frac{1}{\theta^{\overline{\bar{w}}}}-\frac{1}{\theta^{\overline{\overline{w s}}}}\right)
\end{aligned}
$$

and

$$
\begin{aligned}
\stackrel{w s \rightarrow s}{Q}^{\prime} & \left(\frac{E^{\overline{\bar{s}}}}{\epsilon^{s} \rho^{s}}-\mu^{\bar{s}}\right) \stackrel{w s \rightarrow s}{M}-\left\langle\mathbf{n}_{s} \cdot \mathbf{t}_{s} \cdot \mathbf{n}_{s}\right\rangle_{\Omega_{w s}, \Omega_{w s}} \frac{\mathrm{D}^{\bar{s}} \epsilon^{s}}{\mathrm{D} t} \\
& -\left\langle\frac{\boldsymbol{\sigma}_{s}}{\rho_{s}}: \frac{\mathbf{C}_{s}}{j_{s}}-\frac{1}{\rho_{s}} \mathbf{n}_{s} \cdot \mathbf{t}_{s} \cdot \mathbf{n}_{s}\right\rangle_{\Omega_{w s}, \Omega_{w s}} \stackrel{w s \rightarrow s}{M}=\hat{k}_{q}^{s}\left(\frac{1}{\theta^{\bar{s}}}-\frac{1}{\theta^{\overline{w s}}}\right)
\end{aligned}
$$

where $\hat{k}_{q}^{w}$ and $\hat{k}_{q}^{s}$ are non-negative interfacial heat transfer parameters.

\section{Closed Model}

The conservation equations given in $\S 4$ can be combined with the closure relations given in $\S 9$ and some additional assumptions to derive closed models 
for single-phase flow. In the interest of clarity, we will note all assumptions needed to arrive at the closed model in the present study. The model we will derive is a simplified single-phase flow model that we will compare and contrast with the traditional model used to describe such systems.

\subsection{Model Assumptions}

Assumptions in addition to those made previously in the derivation are required to obtain a closed, solvable model that describes macroscale transport phenomena in a porous medium system. The forms and appropriateness of these assumptions will depend upon the physical system of interest. All of the assumptions are subject to revision if the model derived proves inadequate for a particular system whose behavior is not consistent with the assumptions. We summarize the assumptions made to this point and the assumptions we will make going forward to derive the specific model of concern in this paper.

Assumption 1 (Deterministic Macroscale Averaging) A discrete macroscopic length scale exists such that all macroscale quantities of concern are well defined and insensitive to the size of a single representative elementary volume that applies for the deterministic models derived.

Assumption 1 is a standard assumption needed to produced deterministic macroscale models based upon a clear separation of length scales. If a clear separation of length scales does not exist, the fundamental averaging operators and theorems relied upon in this work would need to be revisited.

Assumption 2 (Classical Irreversible Thermodynamics) Classical irreversible thermodynamics is applicable to the porous medium systems of interest 
in this work at the microscale.

Assumption 2 fulfills the need within the TCAT approach to choose a thermodynamic approach. We believe the selection of CIT is reasonable. Additionally, the assumption is made at the microscale, and the consequences of this choice are rigorously established by averaging to the macroscale.

Assumption 3 (Simplified Entropy Inequality) The four approximations used to produce the SEI are: (1) the geometric tensor is independent of entity measures, densities, velocities, interfacial tension, and entropy, such that integrals of products of these quantities may be split into products of integrals; (2) changes in entity measures are not independent and can be approximated using averaging theorems; (3) the systems of concern are macroscopically simple; and (4) terms involving products of microscale quantities where both factors vanish at equilibrium are negligible if both factors are related to solid-phase deformation.

Assumption 3 summarizes a set of conditions imposed to produce the SEI upon which the closure relations are based. We believe this assumption is a reasonable first approximation, and it is testable based upon microscale simulations.

Assumption 4 (Conjugate, First-Order Closure) Valid closure relations for fluxes can be derived from flux-force pairs in the SEI considering only conjugate forces and limiting the approximations to at most first-order Taylor series approximations.

Assumption 4 is a statement of the approach used to develop approximate closure relations used here to produce a closed model. Less restrictive alter- 
natives to this assumption exist and can be employed, as needed, to describe certain systems (e.g., high velocity flow, systems in which coupled processes are important).

Assumption 5 (Isothermal, Linear Compressibility) The system of concern: is isothermal; has negligible interfacial effects; is void of mass exchange between entities; is described by standard equations of state relating mass densities to fluid pressures; has mass densities that have small spatial gradients; has insignificant local and advective acceleration of momentum at the macroscopic scale; contains a solid phase that is linearly compressible, elastic, isotropic, only slightly deformed and solely as a result of a normal stress, and primarily deformed in the vertical direction with small spatial gradients in such deformations; has identical water phase pressures for both volume and area averages; and has integral material derivative fluctuation terms relating microscale and macroscale quantities and arising in the Gibbs-Duhem equation that can be neglected.

Assumption 5 specifies a specific isothermal system for which a model is desired. Several of the specific aspects of this assumption are related to the behavior of the solid phase, and these aspects will be used to arrive at an expression for the solid phase stress tensor that supplements the closure relations derived in $\S 9$.

\subsection{Model Formulation}

The purpose of this section is to produce a complete, closed, and solvable model in terms of measurable parameters and macroscopic variables. The 
model is based upon the conservation equations given in $\S 4$, the closure relations given in $\S 9$, and the assumptions summarized in $\S 10.1$. The target model can be viewed as a parallel to the traditional, isothermal single-phase flow model that is standard in groundwater hydrology [e.g. 4, 11, 12, 14].

The steps involved in producing the target model include (1) specifying the appropriate set of conservation equations, (2) substituting in the closure relations previously developed, (3) applying Assumption 5, (4) determining the additional closure relations needed, and (5) assembling the individual components into a closed model. We detail these steps below.

Based upon this approach, the two conservation of mass equations for the phase entities are of the form

$$
\frac{\mathrm{D}^{\bar{\imath}}\left(\epsilon^{\iota} \rho^{\iota}\right)}{\mathrm{D} t}=-\epsilon^{\iota} \rho^{\iota} \nabla \cdot \mathbf{v}^{\bar{\iota}}, \quad \text { for } \iota \in \mathcal{J}_{p}
$$

The conservation of momentum equation for the $w$ entity is

$$
\frac{\mathrm{D}^{\bar{c}}\left(\epsilon^{w} \rho^{w} \mathbf{v}^{\bar{w}}\right)}{\mathrm{D} t}=-\epsilon^{w} \rho^{w} \mathbf{v}^{\bar{w}} \nabla \cdot \mathbf{v}^{\bar{w}}-\hat{\mathbf{R}}^{w} \cdot \mathbf{v}^{\bar{w}, \bar{s}}-\epsilon^{w} \rho^{w} \nabla\left(\psi^{\bar{w}}+\mu^{\bar{w}}\right)
$$

and for the $s$ entity is

$$
\begin{aligned}
& \frac{\mathrm{D}^{\bar{\iota}}\left(\epsilon^{s} \rho^{s} \mathbf{v}^{\bar{s}}\right)}{\mathrm{D} t}=-\epsilon^{s} \rho^{s} \mathbf{v}^{\bar{s}} \nabla \cdot \mathbf{v}^{\bar{s}}+\nabla \cdot\left(\epsilon^{s} \mathbf{t}^{\overline{\bar{s}}}\right)+\epsilon^{s} \rho^{s} \mathbf{g}^{\bar{s}}+\epsilon^{w} \rho^{w} \mathbf{g}^{\bar{w}} \\
& +\epsilon^{w} \rho^{w} \nabla\left(\psi^{\bar{w}}+\mu^{\bar{w}}\right)-\nabla\left(\epsilon^{w} p^{w}\right)+\hat{\mathbf{R}}^{w} \cdot \mathbf{v}^{\bar{w}, \bar{s}}
\end{aligned}
$$

The mass and momentum equations for the $w s$ interface are ignored as a result of Assumption 5. Also as a result of this assumption, the local and advective acceleration terms in the momentum equation can be ignored giving

$$
\hat{\mathbf{R}}^{w} \cdot \mathbf{v}^{\bar{w}, \bar{s}}=-\epsilon^{w} \rho^{w} \nabla\left(\psi^{\bar{w}}+\mu^{\bar{w}}\right)
$$


and the solid-phase momentum equation

$$
\begin{aligned}
\nabla \cdot & \left(\epsilon^{s} \mathbf{t}^{\overline{\bar{s}}}\right)=-\epsilon^{s} \rho^{s} \mathbf{g}^{\bar{s}}-\epsilon^{w} \rho^{w} \mathbf{g}^{\bar{w}} \\
& -\epsilon^{w} \rho^{w} \nabla\left(\psi^{\bar{w}}+\mu^{\bar{w}}\right)+\nabla\left(\epsilon^{w} p^{w}\right)-\hat{\mathbf{R}}^{w} \cdot \mathbf{v}^{\bar{w}, \bar{s}}
\end{aligned}
$$

or

$$
\nabla \cdot\left(\epsilon^{s} \mathbf{t}^{\bar{s}}\right)=-\epsilon^{s} \rho^{s} \mathbf{g}^{\bar{s}}-\epsilon^{w} \rho^{w} \mathbf{g}^{\bar{w}}+\nabla\left(\epsilon^{w} p^{w}\right)
$$

which can be written in terms of the total stress tensor

$$
\nabla \cdot \mathbf{t}^{\overline{\bar{T}}}=\nabla \cdot\left(\epsilon^{w} \mathbf{t}^{\bar{w}}+\epsilon^{s} \mathbf{t}^{\overline{\bar{s}}}\right)=-\left(\epsilon^{w} \rho^{w} \mathbf{g}^{\bar{w}}+\epsilon^{s} \rho^{s} \mathbf{g}^{\bar{s}}\right)
$$

Because the system is assumed to be isothermal, specification and closure of the energy equations is not required. Model parameters will still depend upon temperature, but the temperature will be assumed to be specified and constant in space and time and identical for all entities.

At this point, the model consists of eight conservation equations, two for mass and six for momentum, that must be solved for the 18 unknowns remaining in the formulation: $\epsilon^{w}, \epsilon^{s}, \rho^{w}, \rho^{s}, \mathbf{v}^{\bar{w}}, \mathbf{v}^{\bar{s}}, \mu^{\bar{w}}, p^{w}$ and $\mathbf{t}^{\overline{\bar{s}}}$. The closure of this model will be accomplished by: (1) using a constraint on the sum of volume fractions, (2) relating mass densities to the fluid pressure through equations of state, (3) using an approximation based upon the Gibbs-Duhem equation to relate the fluid pressure to the chemical potential, and (4) approximating the solid-phase stress tensor, which will be used to compute the solid-phase velocity. These steps are summarized in turn.

The volume fraction constraint is

$$
\epsilon^{w}+\epsilon^{s}=1
$$


Eqn (84) for the $w$ and $s$ entities can be written using eqn (91) as

$$
\frac{\epsilon^{w}}{\rho^{w}} \frac{\partial \rho^{w}}{\partial t}+\frac{\partial \epsilon^{w}}{\partial t}=-\nabla \cdot\left(\epsilon^{w} \mathbf{v}^{\bar{w}}\right)-\frac{\epsilon^{w}}{\rho^{w}} \mathbf{v}^{\bar{w}} \cdot \nabla \rho^{w}
$$

and

$$
\frac{\left(1-\epsilon^{w}\right)}{\rho^{s}} \frac{\partial \rho^{s}}{\partial t}-\frac{\partial \epsilon^{w}}{\partial t}=-\nabla \cdot \mathbf{v}^{\bar{s}}+\nabla \cdot\left(\epsilon^{w} \mathbf{v}^{\bar{s}}\right)-\frac{\left(1-\epsilon^{w}\right)}{\rho^{s}} \mathbf{v}^{\bar{s}} \cdot \nabla \rho^{s}
$$

Summation of these expansions and dropping the spatial gradients of density as specified in Assumption 5 yields

$$
\frac{\epsilon^{w}}{\rho^{w}} \frac{\partial \rho^{w}}{\partial t}+\frac{\left(1-\epsilon^{w}\right)}{\rho^{s}} \frac{\partial \rho^{s}}{\partial t}=-\nabla \cdot\left(\epsilon^{w} \mathbf{v}^{\bar{w}, \bar{s}}\right)-\nabla \cdot \mathbf{v}^{\bar{s}}
$$

Since the system being considered is isothermal and of uniform composition, equations of state for density can be approximated as

$$
\frac{1}{\rho^{w}} \frac{\partial \rho^{s}}{\partial p^{w}}=\hat{\beta}^{w}
$$

and

$$
-\frac{1}{\rho^{s}} \frac{\partial \rho^{s}}{\partial\left(\left\langle\mathbf{n}_{s} \cdot \mathbf{t}_{s} \cdot \mathbf{n}_{s}\right\rangle_{\Omega_{w s}, \Omega_{w s}}\right)}=\hat{\beta}^{s}
$$

where $\hat{\beta}^{w}$ and $\hat{\beta}^{s}$ are compressibility parameters.

Combining eqns (94)-(96) and assuming deformation results from the normal stress alone, we obtain:

$$
\left[\epsilon^{w} \hat{\beta}^{w}+\left(1-\epsilon^{w}\right) \hat{\beta}^{s}\right] \frac{\partial p^{w}}{\partial t}=-\nabla \cdot\left(\epsilon^{w} \mathbf{v}^{\bar{w}, \bar{s}}\right)-\nabla \cdot \mathbf{v}^{\bar{s}}
$$

Substitution of eqn (87) into eqn (97) gives

$$
\left[\epsilon^{w} \hat{\beta}^{w}+\left(1-\epsilon^{w}\right) \hat{\beta}^{s}\right] \frac{\partial p^{w}}{\partial t}=\nabla \cdot\left[\rho^{w} \hat{\mathbf{K}} \cdot \nabla\left(\psi^{\bar{w}}+\mu^{\bar{w}}\right)\right]-\nabla \cdot \mathbf{v}^{\bar{s}}
$$

where the hydraulic conductivity tensor is defined as

$$
\hat{\mathbf{K}}=\left(\epsilon^{w}\right)^{2}\left(\hat{\mathbf{R}}^{w}\right)^{-1}
$$


Application of the Gibbs-Duhem equation while ignoring the fluctuation integral terms arising from averaging from the microscale to the macroscale gives

$$
\left[\epsilon^{w} \hat{\beta}^{w}+\left(1-\epsilon^{w}\right) \hat{\beta}^{s}\right] \frac{\partial p^{w}}{\partial t}=\nabla \cdot\left[\hat{\mathbf{K}} \cdot\left(\nabla p^{w}+\rho^{w} \nabla \psi^{\bar{w}}\right)\right]-\nabla \cdot \mathbf{v}^{\bar{s}}
$$

where the term inside the divergence operator is a consistent averaged form of Darcy's law [19]. Eqn (100) is nearly a result that can be compared with the traditional model, except some additional information is needed regarding $\nabla \cdot \mathbf{v}^{\bar{s}}$. Derivation of such an expression requires consideration of solid mechanics.

Decompose the solid phase stress tensor into component parts

$$
\epsilon^{s} \mathbf{t}^{\overline{\bar{s}}}=-\epsilon^{s} p^{\overline{\bar{s}} \mathbf{I}}+\epsilon^{s} \boldsymbol{\tau}^{\overline{\bar{s}}}
$$

where $p^{\overline{\bar{s}}}$ is a solid-phase pressure and $\tau^{\overline{\bar{s}}}$ is an effective solid-phase stress tensor.

Define the total pressure as

$$
p^{\overline{\bar{T}}}=\epsilon^{s} p^{\overline{\bar{s}}}+\epsilon^{w} p^{w}
$$

and note that taking the $\operatorname{Tr}\left(\mathbf{t}^{\overline{\bar{T}}}\right)$ leads to

$$
\frac{\mathbf{t}^{\overline{\overline{\mathrm{T}}}}: \mathbf{I}}{3}=-p^{\overline{\bar{T}}}+\frac{\epsilon^{s} \boldsymbol{\tau}^{\overline{\bar{s}}}: \mathbf{I}}{3}
$$

Normalizing eqn (103) by $\left\langle\mathbf{n}_{s} \cdot \mathbf{t}^{\overline{\bar{s}}} \cdot \mathbf{n}_{s}\right\rangle_{\Omega_{w s}, \Omega_{w s}}$ yields

$$
\frac{\mathbf{t}^{\overline{\bar{T}}}: \mathbf{l}}{3\left\langle\mathbf{n}_{s} \cdot \mathbf{t}^{\overline{\bar{s}}} \cdot \mathbf{n}_{s}\right\rangle_{\Omega_{w s}, \Omega_{w s}}}=-\frac{p^{\overline{\bar{T}}}}{\left\langle\mathbf{n}_{s} \cdot \mathbf{t}^{\overline{\bar{s}}} \cdot \mathbf{n}_{s}\right\rangle_{\Omega_{w s}, \Omega_{w s}}}+\frac{\epsilon^{s} \boldsymbol{\tau}^{\overline{\bar{s}}}: \mathbf{l}}{3\left\langle\mathbf{n}_{s} \cdot \mathbf{t}^{\overline{\bar{s}}} \cdot \mathbf{n}_{s}\right\rangle_{\Omega_{w s}, \Omega_{w s}}}
$$

The left hand side of this equation amounts to a ratio of forces. For the case of small, slow deformations this ratio is 1 to a good approximation. The first 
term on the right hand side of eqn (104) is defined as the Biot coefficient, $\hat{\alpha}$, giving

$$
1=\hat{\alpha}+\frac{\epsilon^{s} \boldsymbol{\tau}^{\overline{\bar{s}}}: \mathbf{I}}{3\left\langle\mathbf{n}_{s} \cdot \mathbf{t}^{\overline{\bar{s}}} \cdot \mathbf{n}_{s}\right\rangle_{\Omega_{w s}, \Omega_{w s}}}
$$

From Assumption 5 the solid is linear, elastic, and isotropic and subject to small, slow deformations, which allows the effective solid phase stress tensor to be written as

$$
\epsilon^{s} \boldsymbol{\tau}^{\overline{\bar{s}}}=\frac{\hat{\nu} \hat{E}}{(1-2 \hat{\nu})(1+\hat{\nu})} \mathbf{e}^{s}: \mathbf{I}+\frac{\hat{E}}{1+\hat{\nu}} \mathbf{e}^{s}
$$

where $\mathbf{e}^{s}$ is an Eulerian strain tensor, $\hat{\nu}$ is Poisson's ratio, and $\hat{E}$ is Young's modulus.

The trace of eqn (106) is

$$
\epsilon^{s} \boldsymbol{\tau}^{\overline{\bar{s}}}: \mathbf{I}=\frac{\hat{E}}{(1-2 \hat{\nu})} \mathbf{e}^{s}: \mathbf{I}=3 \hat{K}_{T} \mathbf{e}^{s}: \mathbf{I}
$$

which allows for

$$
\hat{\alpha}=1-\frac{\hat{K}_{T} \mathbf{e}^{s}: \mathbf{l}}{\left\langle\mathbf{n}_{s} \cdot \mathbf{t}_{s} \cdot \mathbf{n}_{s}\right\rangle_{\Omega_{w s}, \Omega_{w s}}}=1-\frac{\hat{K}_{T}}{\hat{K}_{S}}
$$

where $\hat{K}_{T}$ is the bulk modulus of the skeleton and $\hat{K}_{S}$ is the bulk modulus of the solid phase. The solid phase bulk modulus is equal to the inverse of the solid compressibility such that

$$
\hat{\alpha}=1-\frac{\hat{K}_{T}}{\hat{K}_{S}}=1-\hat{\beta}^{s} \hat{K}_{T}
$$

Combining results, we obtain the total stress tensor

$$
\mathbf{t}^{\overline{\bar{T}}}=\hat{\alpha}\left\langle\mathbf{n}_{s} \cdot \mathbf{t}_{s} \cdot \mathbf{n}_{s}\right\rangle_{\Omega_{w s}, \Omega_{w s}} \mathbf{I}+\epsilon^{s} \boldsymbol{\tau}^{\overline{\bar{s}}}
$$

Since interfacial tension effects are negligible, changes in porosity are slow, and the volume averaged pressure is assumed equal to the average of the pressure 
over the interfacial area then

$$
\mathbf{t}^{\overline{\bar{T}}}=-\hat{\alpha} p^{w} \mathbf{I}+\frac{\hat{\nu} \hat{E}}{(1-2 \hat{\nu})(1+\hat{\nu})} \mathbf{e}^{s}: \mathbf{I}+\frac{\hat{E}}{1+\hat{\nu}} \mathbf{e}^{s}
$$

From Assumption 5, deformation is assumed to primarily occur in the vertical dimension; and horizontal spatial gradients of this deformation are assumed to be negligible. This allows for further simplification of the total stress tensor to

$$
\mathbf{t}^{\overline{\bar{T}}}=-\hat{\alpha} p^{w} \mathbf{I}+\frac{\hat{\nu} \hat{E}}{(1-2 \hat{\nu})(1+\hat{\nu})} e_{z z}^{s} \mathbf{I}+\frac{\hat{E}}{1+\hat{\nu}} e_{z z}^{s} \mathbf{k} \mathbf{k}
$$

Since deformation is primarily in the vertical dimension, eqn (112) can be differentiated with respect to $z$ and equated with the vertical component of eqn (90) giving

$$
\frac{\partial\left(\hat{\alpha} p^{w}\right)}{\partial z}-\frac{\partial\left(\hat{E}_{\nu} e_{z z}^{s}\right)}{\partial z}=-\rho^{\mathrm{b}} g
$$

where

$$
\hat{E}_{\nu}=\frac{(1-\hat{\nu}) \hat{E}}{(1-2 \hat{\nu})(1+\hat{\nu})}=3 \hat{K}_{T} \frac{1-\hat{\nu}}{1+\hat{\nu}}
$$

the bulk density is

$$
\rho^{\mathrm{b}}=\epsilon^{w} \rho^{w}+\epsilon^{s} \rho^{s}
$$

and $g$ is the magnitude of the gravitational acceleration, which acts in the $-\mathbf{k}$ direction.

Integration of eqn (113) with respect to the vertical dimension, assuming $\rho^{\mathrm{b}}$ is a constant, and differentiation of this expression with respect to time yields

$$
\frac{\partial e_{z z}^{s}}{\partial t}=\frac{\hat{\alpha}}{\hat{E}_{\nu}} \frac{\partial p^{w}}{\partial t}
$$

We need to relate the time rate of change of the strain given by eqn (116) to the divergence of solid-phase velocity to complete the closure of the model. 
Because of the small deformation assumption, the distinction between the Eulerian and Lagrangian strains disappears [13]. With the assumption that the spin tensor is small because of deformation primarily in the vertical direction, then

$$
\left\langle\frac{\partial \mathbf{e}_{s}}{\partial t}\right\rangle_{\Omega_{s}, \Omega}=\left\langle\mathbf{d}_{s}\right\rangle_{\Omega_{s}, \Omega}
$$

Application of the averaging theorems [18] to eqn (117) to convert the averages of integrals to the integrals of averages gives

$$
\frac{\partial\left(\epsilon^{s} \mathbf{e}^{s}\right)}{\partial t}-\left\langle\mathbf{e}_{s} \mathbf{v}_{w s} \cdot \mathbf{n}_{s}\right\rangle_{\Omega_{w s}, \Omega}-\frac{1}{2}\left\{\nabla\left(\epsilon^{s} \mathbf{v}^{\bar{s}}\right)+\left[\nabla\left(\epsilon^{s} \mathbf{v}^{\bar{s}}\right)\right]^{\mathrm{T}}\right\}=\frac{1}{2}\left\langle\mathbf{n}_{s} \mathbf{v}_{s}+\mathbf{v}_{s} \mathbf{n}_{s}\right\rangle_{\Omega_{s}, \Omega}
$$

Dotting eqn (118) with I allows for

$$
\epsilon^{s} \frac{\partial \mathbf{e}^{s}: \mathbf{I}}{\partial t}-\epsilon^{s} \nabla \cdot \mathbf{v}^{\bar{s}}=-\mathbf{e}^{s}: \mathbf{I} \frac{\partial \epsilon^{s}}{\partial t}+\mathbf{v}^{\bar{s}} \cdot \nabla \epsilon^{s}+\left\langle\left[\left(\mathbf{e}_{s}: \mathbf{I}\right) \mathbf{v}_{w s}+\mathbf{v}_{s}\right] \cdot \mathbf{n}_{s}\right\rangle_{\Omega_{w s}, \Omega}
$$

Because of Assumption 5, including slow deformations with negligible spatial gradients and interfacial effects, the terms on the right-hand-side of eqn (119) are negligible. Also, since the dominant strain tensor term is in the vertical dimension, we can write eqn (119) as

$$
\frac{\partial e_{z z}^{s}}{\partial t}-\nabla \cdot \mathbf{v}^{\bar{s}}=0
$$

Combining eqns (116) and (120) we obtain

$$
\frac{\hat{\alpha}}{\hat{E}_{\nu}} \frac{\partial p^{w}}{\partial t}-\nabla \cdot \mathbf{v}^{\bar{s}}=0
$$

which may be used along with eqn (100) to yield a closed model for singlephase flow

$$
\left[\epsilon^{w} \hat{\beta}^{w}+\left(1-\epsilon^{w}\right) \hat{\beta}^{s}+\frac{\hat{\alpha}}{\hat{E}_{\nu}}\right] \frac{\partial p^{w}}{\partial t}=\nabla \cdot\left[\hat{\mathbf{K}} \cdot\left(\nabla p^{w}+\rho^{w} \nabla \psi^{\bar{w}}\right)\right]
$$


For the case in which the change in $\epsilon^{w}$ with time is neglected, this equation is a closed model. It is also interesting to note that application of the Gibbs-Duhem equation while recalling the stipulation that spatial gradients of density can be neglected allows eqn (122) to be written as

$$
\left[\epsilon^{w} \hat{\beta}^{w}+\left(1-\epsilon^{w}\right) \hat{\beta}^{s}+\frac{\hat{\alpha}}{\hat{E}_{\nu}}\right] \frac{\partial \mu^{\bar{w}}}{\partial t}=\nabla \cdot\left[\hat{\mathbf{K}} \cdot \nabla\left(\mu^{\bar{w}}+\psi^{\bar{w}}\right)\right]
$$

\subsection{Model Comparison}

The traditional single-phase groundwater flow model is [e.g. 4, 11, 12, 14]

$$
\hat{S}_{s} \frac{\partial H}{\partial t}=\nabla \cdot(\hat{\mathbf{k}} \cdot \nabla H)
$$

where $\hat{S}_{s}$ is the specific storage parameter and $H$ is the hydraulic head.

Eqn (122) can be transformed into an identical form as eqn (124) by expressing the combination of pressure head and gravitational potential as hydraulic head and approximating the left hand side as the product of a single lumped coefficient and temporal derivative of hydraulic head. Some additional approximations are involved in such a transformation, but these are part of the derivation of the traditional model and the errors in these further approximations have been noted and bounded [11]. The direct correspondence between eqn (123) and eqn (124) is also apparent when $\psi^{\bar{w}}$ is independent of time. Under the conditions where the transformation applies, the specific storage parameter relates to the parameters in equation eqn (122) as

$$
\hat{S}_{s}=\rho^{w} g\left[\epsilon^{w} \hat{\beta}^{w}+\left(1-\epsilon^{w}\right) \hat{\beta}^{s}+\frac{\hat{\alpha}}{\hat{E}_{\nu}}\right]
$$

Typically, the deformation of the solid grains is considered small relative to 
the deformation of the solid skeleton so that the Biot coefficient, $\hat{\alpha}$, is equal to 1 . In general $\hat{\alpha} / \hat{E}_{\nu}$ may be identified as the compressibility of the porous medium. When $\hat{\alpha}=1$, the result here is consistent with standard formulations $[5]$.

It is comforting to arrive at a single-phase-flow model that is consistent with the traditional model, since the traditional model has proven to be of significant utility for solving many practical problems. However, eqn (122) does have some advantages compared to the traditional model. First, eqn (122) is expressed in terms of precisely defined variables that have been rigorously connected to the microscale. Second, the many assumptions needed to arrive at this model have been clearly expressed. Third, the foundation has been laid to relax these assumptions and produce alternative models, which will be needed to describe systems for which the assumptions made do not result in sufficiently accurate models.

Consider the case of precisely described variables. Lacking such a definition, it is impossible to rigorously couple microscale simulations or theory to the macroscale or to design or interpret macroscale measurements that one can be assured do not include an implicit source of error. As an example, it is standard practice to average the traditional model over the vertical dimension. If such averaging is not done with care in terms of rigorously defined quantities, paradoxes can arise, as was shown in a recent analysis of Darcy's law by [19].

Careful and complete specification of assumptions needed to arrive at a model are an important prerequisite to appropriate application of that model. Since models are simplifications of reality, it is crucial to understand the nature of these simplifications in detail so that conditions for which the assumptions 
made are not applicable can be identified, hopefully a priori.

Having a foundation in place to relax assumptions and produce alternative models is important as well. Such a foundation forms the basis for evaluating errors made when assumptions are not met and provides a means to produce more reliable descriptions of situations in which assumptions associated with the traditional model are not appropriate.

\section{Discussion}

Three items warrant a brief discussion: (1) the general nature of the approach developed and illustrated through this work, (2) specific extensions to this work on modeling single-phase flow that are possible, and (3) some specific considerations involved with the extension of this general approach to other sorts of systems. We comment on each of these items in turn.

While the general steps involved in the TCAT approach were presented in the first paper in this series [20], a primary objective of this work was to detail the TCAT approach for a specific application. We chose a relatively simple example for this demonstration so that the steps involved could be clearly presented without getting lost in details. Aspects of this work that are novel, and we believe important, include the selection of a microscale thermodynamic theory and averaging of this theory to the macroscale; the formulation of equilibrium conditions; the use of equilibrium conditions to inform the EPP; the Lagrange multiplier approach used to constrain the EI; the separation of the exact form of the CEI, given the thermodynamic basis chosen, from the approximate form of the SEI; the resultant flux-force form 
of the SEI that corresponds to the equilibrium conditions and the EPP; and the approach for producing a closed and solvable model. The general TCAT approach, as well as the specific aspects of the application of this approach shown in this work, can be extended to many other types of systems. Such extensions include systems in which the composition of the phases is subject to changes and multiple fluid phase systems, both with and without composition effects. Future work in this series will focus on producing hierarchies of models for these other classes of applications.

The class of problems considered, single-phase flow, is relatively simple and the specific closed model developed is an especially simple instance of a model from this class. A strength of the TCAT approach is that a complete, explicit list of assumptions needed to produce this model instance is available and as are the means to produce alternative models based upon a different or relaxed set of assumptions. Even within this relatively simple class of models, many other model instances are possible. Rather than attempt to detail a long list of such model instances, we will consider the explicit assumptions made in arriving at the specific closed model formulated in this work and comment on the consequences arising from alternative choices that could me made for each of these assumptions.

Assumption 1 requires a clear separation of length scales that may not exist for many natural systems. The models derived based upon this assumption are deterministic in form. Many would view this as a reasonable starting point for fundamental work of this sort. The extension of these models to a stochastic form follows naturally from the resultant deterministic models by allowing for macroscale parameters and auxiliary conditions to be stochastic in nature while the model form is consistent with the deterministic form; this 
is a commonly used approach. However as an alternative to this, the single REV assumption could be relaxed to include REV's that vary as a function of quantity being considered or even to the stochastic case where averaging from the microscale is considered in a stochastic sense. Both of these approaches warrant additional consideration.

Assumption 2 was needed to connect the conservation equations to the entropy inequality. The approach taken in this work represents the first example of macroscale model formulation and closure based upon microscale thermodynamics that was averaged to the macroscale. A result of this approach is explicit expressions for fluctuation quantities neglected in standard models such that the importance of these terms can be assessed. Alternative thermodynamic approaches exist, and some of these approaches were reviewed previously [20]. For cases in which CIT must be extended such that thermodynamics are consistent with observations, that extended thermodynamic approach can be incorporated into the present framework at the microscale and then averaged to the macroscale. The averaging of such a framework to the macroscale would follow a similar approach to that used here.

Assumption 3 was made to simplify the geometric aspects of the SEI and ultimately influenced the form of the closure relations. Because interfacial effects were later neglected, this assumption is of minimal importance for the specific model produced in this work. If one wished to advance models that included interfacial effects, a closer examination of this assumption would be warranted. Geometric assumptions are expected to occur for more complex systems as well and to be of relatively greater importance in multiple fluid phases cases especially when interface dynamics influence system behavior. This assumption also included the macroscale simple system requirement. If 
this turns out to be an invalid assumption in certain situations, then closure approximations could be developed using approaches similar to those used for the force-flux pairs. Finally, cases in which large deformations of the solid phase occur would require reexamination of the assumption that the solid phase is in a quasi-equlibrium state, which allowed us to drop the higher order product terms associated with solid deformation.

Assumption 4 is known to be an approximation that does not hold in general. For example, fluxes are well-known to depend on forces in addition to their conjugate forces. This dependence can be observed explicitly from the SEI that was derived. The assumption of a low-order approximation is also wellknown to be overly restrictive in certain cases. For example, the literature is replete with observations of flows that are not well described by Darcy's law, and alternative models have been developed and evaluated using experimental and microscale simulation approaches. Many unresolved issues remain for both of these extensions to the uncoupled, low-order theory used in this work.

Assumption 5 is an explicit statement of a very simple single-phase-flow case, yet it is consistent with a model traditionally used in practice. Relaxing some of these assumptions would be necessary to describe systems for which significant consolidation occurs. Further, fluid flow in non-isothermal porous medium systems is an area of active research with many unresolved questions. Both consolidation and non-isothermal systems warrant further consideration and the approach developed in this work provides a framework for obtaining improved descriptions of these processes.

Finally, the TCAT approach can be used to formulate models that are significantly more complicated than the single-phase-flow model. These models may 
include not only the extensions noted above, but also cases in which multiple fluid phases are considered. For such cases, the role of interfaces will become increasingly important and these entities will need to be considered to represent most systems of concern. More complex systems will also lead to a need for nonlinear closure relations. Development of these relations at the macroscale can be aided by microscale analyses that is averaged to the macroscale. The explicit relations between microscale and macroscale variables makes TCAT a useful framework for interrelating microscale and macroscale experiments and theory.

\section{Conclusions}

This work details the elements of the TCAT approach for constructing models of multiphase porous medium systems. Novel aspects of this work include the rigorous treatment of thermodynamics, the development and use of equilibrium conditions to guide the formulation of a simplified entropy inequality, and the use of a concise Lagrange multiplier approach to connect the conservation equations to the entropy inequality. A clear separation of exact results from approximations, which are both needed to produced closed models and explicitly annotated, is included.

The TCAT approach is combined with a set of assumptions needed to produce a model that is similar to the traditional single-phase-flow model. However, the resultant model is defined in terms of precise quantities that are firmly connected to the microscale. The path forward from this simple model to more complex cases is discussed and arises directly from the formulation. Potential single-phase-flow extensions that could be considered are detailed and include 
systems for which consolidation is important, non-isothermal systems, and systems for which cross-coupling and non-Darcy flow regimes are of interest.

The TCAT approach can be applied to more complicated systems involving multiple fluid phases and multiple species. While the general TCAT approach will be similar for these systems, some important differences are noted. Specifically, interfacial effects will be important and a reliance will be made on microscale analysis to produce the specific form of closure relations needed to yield closed, solvable models.

\section{Acknowledgments}

This work was supported in part by National Science Foundation grants DMS0327896 and EAR-0337535 and by National Institute of Environmental Health Sciences grant P42 ES05948.

\section{References}

[1] J. I. D. Alexander and W. C. Johnson. Thermomechanical equilibrium in solid-fluid systems with curved interfaces. Journal of Applied Physics, 58(2):816-824, 1985.

[2] J. Barrere, O. Gipouloux, and S. Whitaker. On the closure problem for Darcy's law. Transport in Porous Media, 7:209-222, 1992.

[3] J. Bear. Dynamics of Fluids in Porous Media. Elsevier, New York, 1972.

[4] J. Bear. Hydraulics of Groundwater. McGraw-Hill, New York, 1979.

[5] J. Bear and Y. Bachmat. Introduction to Modeling of Transport Phe- 
nomena in Porous Media. Kluwer Academic Publishers, Dordrecht, The Netherlands, 1991.

[6] L. Boruvka. An Extension to Classical Theory of Capillarity. Masters thesis, University of Toronto, Toronto, 1975.

[7] L. Boruvka and A. W. Neumann. Generalization of the classical theory of capillarity. Journal of Chemical Physics, 66(12):5464-5476, 1977.

[8] H. B. Callen. Thermodynamics and an Introduction to Thermostatistics. Wiley, New York, 1985.

[9] H. Darcy. Les Fontaines Publiques de la Ville de Dijon. Dalmont, Paris, 1856.

[10] H. Darcy. Determination of the laws of flow of water through sand. In R. A. Freeze and W. Back, editors, Physical Hydrology. Hutchinson Ross, Stroudsburg, PA, 1983.

[11] G. de Marsily. Quantitative Hydrogeology: Groundwater Hydrology for Engineers. Academic Press, San Diego, CA, 1986.

[12] P. A. Domenico and F. W. Schwartz. Physical and Chemical Hydrogeology. John Wiley and Sons, New York, 1998.

[13] A. C. Eringen. Mechanics of Continua. Krieger, Huntington, NY, 1980.

[14] R. A. Freeze and J. A. Cherry. Groundwater. Prentice-Hall, Inc., Englewood Cliffs, NJ, 1979.

[15] W. G. Gray. Thermodynamics and constitutive theory for multiphase porous-media flow considering internal geometric constraints. Advances in Water Resources, 22(5):521-547, 1999.

[16] W. G. Gray. On the definition of derivatives of macroscale energy for the description of multiphase systems. Advances in Water Resources, 25 (8-12):1091-1104, 2002.

[17] W. G. Gray and S. M. Hassanizadeh. Macroscale continuum mechanics 
for multiphase porous-media flow including phases, interfaces, common lines and common points. Advances in Water Resources, 21(4):261-281, 1998.

[18] W. G. Gray, A. Leijnse, R. L. Kolar, and C. A. Blain. Mathematical Tools for Changing Scales in the Analysis of Physical Systems. CRC Press, Inc., Boca Raton, FL, 1993.

[19] W. G. Gray and C. T. Miller. An examination of Darcy's law for flow in variable porosity porous media. Environmental Science \& Technology, 38 (22):5895-5901, 2004.

[20] W. G. Gray and C. T. Miller. Thermodynamically constrained averaging theory approach for modeling flow and transport phenomena in porous medium systems: 1. Motivation and overview. Advances in Water Resources, 28(2):161-180, 2005.

[21] W. G. Gray and B. A. Schrefler. Analysis of the solid phase stress tensor in multiphse porous media. In review in International Journal for Numerical and Analytical Methods in Geomechanics, 2005.

[22] W. G. Gray, A. F. B. Tompson, and W. E. Soll. Closure conditions for two-fluid flow in porous media. Transport in Porous Media, 47(1):29-65, 2002.

[23] D. Jou, J. Casas-Vazquez, and G. Lebon. Extended Irreversible Thermodynamics. Springer-Verlag, Berlin, 2001.

[24] M. G. McDonald and A. W. Harbaugh. A modular three-dimensional finite-difference groundwater-flow model. Technical report, U.S. Geological Survey, Scientific Publications Co., Washington, D.C., 1988.

[25] C. T. Miller and W. G. Gray. Thermodynamically constrained averaging theory approach for modeling flow and transport phenomena in porous medium systems: 2. Foundation. Advances in Water Resources, 28(2): 
181-202, 2005.

[26] M. Panfilov. Macroscale Models of Flow Through Highly Heterogeneous Porous Media. Springer, 2000.

[27] M. Quintard and S. Whitaker. Transport in ordered and disordered porous media, 3. Closure and comparison between theory and experiment. Transport in Porous Media, 15(1):31-49, 1994.

[28] S. Whitaker. Advances in theory of fluid motion in porous media. Industrial and Engineering Chemistry, 61(12):14-28, 1969.

[29] S. Whitaker. Flow in porous media I: A theoretical derivation of Darcy's law. Transport in Porous Media, 1:3-25, 1986.

[30] S. Whitaker. The method of volume averaging. In J. Bear, editor, Theory and Application of Transport in Porous Media. Kluwer, Dordrecht, Netherlands, 1998. 